Feature

\title{
Computability and Evolutionary Complexity: Markets As Complex Adaptive Systems (CAS) ${ }^{1}$
}

\author{
Sheri M.Markose \\ Economics Department \\ University of Essex \\ Wivenhoe Park \\ Colchester C04 3SQ \\ Essex, UK \\ Email: scher@essex.ac.uk \\ September 2003
}

\begin{abstract}
The purpose of this Feature is to critically examine and to contribute to the burgeoning multi disciplinary literature on markets as complex adaptive systems (CAS). Three economists, Robert Axtell, Steven Durlauf and Arthur Robson who have distinguished themselves as pioneers in different aspects of how the thesis of evolutionary complexity pertains to market environments have contributed to this special issue. Axtell is concerned about the procedural aspects of attaining market equilibria in a decentralized setting and argues that principles on the complexity of feasible computation should rule in or out widely held models such as the Walrasian one. Robson puts forward the hypothesis called the Red Queen principle, well known from evolutionary biology, as a possible explanation for the evolution of complexity itself. Durlauf examines some of the claims that have been made in the name of complex systems theory to see whether these present testable hypothesis for economic models. My overview aims to use the wider literature on complex systems to provide a conceptual framework within which to discuss the issues raised for Economics in the above contributions and elsewhere. In particular, some assessment will be made on the extent to which modern complex systems theory and its application to markets as $\mathbf{C A S}$ constitutes a paradigm shift from more mainstream economic analysis.
\end{abstract}

\footnotetext{
${ }^{1}$ I am grateful for discussions over the years with Ken Binmore, Steve Spear, Vela Velupillai and for an important meeting and email exchanges with Herbert Simon. Bernhard von Stengel kindly gave detailed comments on Complexity of Exchange and John Sutton has been very generous to me with his data that has been used in Section 3. I'm grateful to Eliot Maenner for improving the aesthetic quality of the paper tremendously. Recent discussions with Thomas Lux, Jasmina Arifovic, Edward Tsang and the Feature contributors Robert Axtell, Steven Durlauf and Arthur Robson have helped produce a coherent whole. I particularly appreciate Steve Machin's encouragement and patience in the process of bringing this Feature to fruition.
} 


\section{Computability and Evolutionary Complexity: Markets As Complex Adaptive Systems (CAS) Sheri M. Markose}

Few will contest that epi-phenomena of biological systems and socio- economic systems is anything but complex. The purpose of this Feature is to critically examine and to contribute to the burgeoning multi disciplinary literature on markets as complex adaptive systems (CAS). The new sciences of complexity, the principles of self-organization and emergence along with the methods of evolutionary computation and artificially intelligent agent models have been developed in a multi-disciplinary fashion. The cognoscenti here view that complex systems whether natural or artificial, physical, biological or socio-economic can be characterized by a unifying set of principles. Further, it is held that these principles mark a paradigm shift from earlier ways of viewing such phenomenon.

Three economists, Robert Axtell, Steven Durlauf and Arthur Robson who have distinguished themselves as pioneers in different aspects of how the thesis of evolutionary complexity pertains to market environments have contributed to the special issue. Axtell is concerned about the procedural aspects of attaining market equilibria in a decentralized setting and argues that principles on the complexity of feasible computation should rule in or out widely held models such as the Walrasian one. Robson puts forward the hypothesis called the Red Queen principle, well known from evolutionary biology, as a possible explanation for the evolution of complexity itself. Durlauf examines some of the claims that have been made in the name of complex systems theory to see whether these present testable hypotheses for economic models. My overview aims to use the wider literature on complex systems to provide a conceptual framework within which to discuss the issues raised for Economics in the above contributions and elsewhere. In particular, some assessment will be made on the extent to which modern complex systems theory and its application to markets as CAS constitutes a paradigm shift from more mainstream economic analysis.

The earliest precursor to modern complex systems theory resides in the classical $18^{\text {th }}$. century political economy of the Scottish Enlightenment that order in market systems is spontaneous or emergent in that it is the result of 'human action and not the execution of human design'. This early observation, well known also from the Adam Smith metaphor of the invisible hand, premises a disjunction between system wide outcomes and the design capabilities of individuals at a micro level and the distinct absence of an external organizing force. It has been claimed that not just market equilibria but many institutions and artifacts in society ranging from language to division of labour, civil society and monetary exchange are unintended consequences of individuals' actions rather than those borne of rational calculation 
and design. This anti creationist classical thesis in Economics which marks the provenance of modern evolutionary thought is also supposed to have predated and influenced Darwin, Hodgson (1993). The paradigm change implied by the anti-creationist thesis in evolution, the so called contra "argument from design" pertains to the most remarkable of macroevolutionary trends viz. the emergence of new forms within more complex organisms or systems.

Section 1 briefly reviews the multi disciplinary and computational legacy of CAS theory. The sine qua non of a CAS will be found to be its capacity to produce novelty or 'surprises' with a mathematically non-trivial definition for the non-anticipating global ordering pattern for the system with interacting constituent elements. The latter achieved in the absence of central command is often referred to as self-organization or emergence of macroscopic properties of the system. In Section 1.2, I will delineate some formal issues regarding the three major perspectives on self-organized complexity and these are not all mutually exclusive to the different sciences involved here, viz. Computer Science/Mathematics, Physics, Biology and Economics. In all variants of complex systems theory it is held that macroscopic properties cannot be formally or analytically deduced from the properties of its parts. Methodologically, it is precisely this that distinguishes the sciences of complex systems from the bulk of traditional science which relies on deductive formalistic and analytical methods. However, as will be seen in Sections 3 and 4, different postulates exist on what are regarded to be the testable and observable aspects of CAS depending on whether or not the emergence of new forms or the capacity for variety is endogenous to the process of self-organization.

It will be argued that it was not well into the 20 th. century with two epochal developments in the foundations of mathematics and advances in computer technology that definitive formulations of CAS are possible. The first of this is the Gödel-Turing-Post ${ }^{2}$ results on incompleteness and algorithmically unsolvable problems where for the first time the logical impossibility limits to formalistic calculation or deductive methods were established. In the absence of these limits on computability there is in principle no reason why creationism or a designing mind is not the force behind all observed patterns and new forms. Indeed, without these foundational advances on computation and incompleteness or what Goldberg ${ }^{3}$ (1995) calls "a heavy dose of mechanism", it is not possible to explain the necessity for the emergence of newly adapted forms which is considered to be the hallmark of CAS. The three major natural exponents of CAS are evolutionary biology, immune systems and innovation based structure changing growth of capitalist systems dubbed creative destruction by Schumpeter (1950). The

\footnotetext{
${ }^{2}$ The seminal papers here are Gödel (1931), Turing (1936) and Post (1944).

${ }^{3}$ Goldberg (1995) claims that the mystery shrouding innovation can be dispelled .. "by a heavy dose of mechanism. Many of the difficulties in the social sciences comes from a lack of a computational theory of actor innovation .... . population oriented systems are dominated by what economists call the law of unintended consequences (which is itself largely the result of the innovative capability of the actors )", (Ibid. p.28).
} 
second significant methodological development is the Holland-Bak-Arthur use of computer based artificial environments to simulate dynamics from large numbers of interacting agents with varying levels of computational and adaptive intelligence to give material counterpart in virtual environments to the otherwise elusive phenomenon of emergence and self-organization.

Though many of these advances in the methodology of science have bypassed mainstream economics (see, Krugman, 1996), the contributions of some economists to CAS theory have been substantial. The seminal work of Schelling (1978) is one of the earliest examples of the use of computer simulation to demonstrate how simple micro behavioural rules result in a self-organized macro outcome, an undesirable one of racial segregation, which could not have been deduced from the initial rules. ${ }^{4}$ More recently, a number of economists and physicists have got involved in the new computational agent based modelling in economics and they have respectively been called ACEs (Adaptive Computational Economists, see Tesfatsion, 1998) and econo-physicists.

A major part of self-organization in markets relies on learning rational expectations equilibria or fixed point mappings of strongly self- referential system wide properties. Section 2 briefly discusses the computational and complexity issues relating to price formation and market equilibria. In Section 2.1, I examine Axtell's intriguing conjecture, this issue, that decentralized arrangements of exchange are also ones that permit feasible real time polynomial complexity in price determination. In Section 2.2 Arthur's famous El Farol game (1994) with its contrarian structure is given to illustrate the fundamental problem of inductive inference in prices in pure speculative markets which militates against homogenous rational expectations.

Section 3 outlines some recent seminal developments on the issues relating to the emergence of new forms and the growth of complexity. Much of this discussion can be subsumed under the Red Queen principle on competitive coevolution that was first discussed in the Economics literature by Robson (2002) and in this Feature. In Section 3.2 some economic examples of the Red Queen such as competitive product innovation, the Lucas (1972) postulates on regulatory arbitrage and on the strategic use of 'surprises' are given. Section 3.3 highlights the significance of oppositional structures or of 'parasites' in the emergence of new forms which becomes a means by which systems escape from entrapment at local optima to global optima. The necessity of 'parasites' or hostile agents is shown to be pertinent both in experimental artificial environments as well as in the formal logic of Gödel (1931) who mechanizes the exit route that leads to innovation from a non-computable fixed point involving the contrarian player, the Liar.

\footnotetext{
${ }^{4}$ In Schelling's racial segregation model, households move residence because of a weak preference for a neighbourhood that has at least one third of those adjoining them to be of the same colour or race. Over time this results in very segregated neighbourhoods though everyone is initially placed at random and nobody is particularly intolerant.
} 
Section 4 covers some ground on the relationship between empirical economics in terms of testable hypotheses of CAS theory. In section 4.1, a brief overview of Durlauf's contribution shows that the two main traditional methods of theory validation with varying degrees of rigour - the historical or case study method and econometric analysis - have for the most part failed to give evidence that can be specifically adduced to CAS. In other words, as other explanations can fit the bill or the power of econometric tests is weak, identification problems loom large. The latter is true also for agent based models. In sections 4.2 and 4.3, the Red Queen effects and a lack of structural invariance and failure of meta (econometric) models to identify strategically induced innovation based structural change are shown to be important additions to the so called stylized CAS facts to do with lock ins, path dependence, network effects, non linearities from thresholds and self-referential calculations, power law distributions, long memory and fat tails. This is followed by a brief concluding section.

\section{Modern Complex Systems Theory}

1.1 The Multi-Disciplinary and Computational Legacy

Pioneering multi disciplinary work in this area, without being exhaustive, has included that of computer scientists and mathematicians ((von Neumann (1970), Wolfram (1984), Penrose (1988), Holland (1975, 1992), Koza (1992) and Goldberg (1989)); physicists (Nicolis and Prigogine (1977, 1989), Bak and Wiesenfeld(1988), Bak (1996), Anderson et. al. (1988), Langton et. al.(1992)); biologists (Kauffman (1993), Green (1994)); and economists (Chen and Day (1993), Axelrod (1984, 1987), Dosi and Nelson (1994), Epstein and Axtell (1996), Krugman (1996), Arthur et. al. (1997), Albin (1998) and Velupillai (2000)). A number of these have been associated with the Santa Fe Institute, USA.

It is the work of John von Neumannn in the 1940's on self-reproducing machines as models for biological systems and self- organized complexity ${ }^{5}$ which provides a landmark transformation of dynamical systems theory based on motion, force and energy to the capabilities and constraints of information processors modelled as computing machines. The von Neumann models based on cellular automata ${ }^{6}$ have laid the ground rules of modern complex systems theory regarding -(i) the use of large ensembles of micro level computational entities or automata following simple rules of local interaction and connectivity, (ii) the capacity of these computational entities to self-reproduce and also to produce automata of greater complexity than themselves and (iii) use of the principles of computing machines to explain diverse system wide or global dynamics.

\footnotetext{
${ }^{5}$ Mirowski(2002) discusses how radical a shift this has been for the methodology of science and also from the perspective of von Neumann's earlier work with Oscar Morgenstern on the Theory of Games and Economic Behaviour .

${ }^{6}$ Cellular automata were developed by von Neumann and Stanislav Ulam to represent biological systems and for the purpose of modelling biological self-reproduction.
} 
The significance of the von Neumann computational legacy of CAS is that it covers all substrata, ranging from the bio-chemical to the artificial, in which effective procedures or computation reside. By the Church-Turing thesis (see, Cutland 1980) the intuitive notion of effective procedures or an algorithm can be identified with the class of general recursive functions and represent finitely encodable programs implemented in a number of equivalent ways referred to as automata or mechanism. The best known among these idealizations of mechanism is the Turing machine and no mechanism can exceed the computational powers of Turing machines. Such a definition of mechanism or formalistic calculation is necessary before complexity measures of the disjunction between the microscopic elements of the system and their macroscopic properties can be ascertained and also on what constitutes an innovation or surprise in the system. Further, there are compelling reasons why the powerful agenda of Herbert Simon $(1956,1978)$ on the procedural lacunae of rationality to further our understanding of observable boundedly rational behaviour, should be securely based on modern computability theory on the limits and efficacy of effective procedures.

In keeping with (i) above, as observed by Arthur (1991), the units of modern adaptive models are "parametrized decision algorithms" or units whose behaviour is brought about by finitely encodeable algorithms. Indeed, as noted by Langton (1992) physical dynamical systems "are bound by the same in principle limitations as computing devices" (ibid.p82). These limitative results of computing devices are generically referred to as the halting problem. Church's Theorem and in particular the Gödel (1931) First Incompleteness Theorem show how Turing machines themselves can produce encoded objects (viz. by mechanizing the exit route in Georg Cantor's famous diagonal method ) that cannot be enumerated by any machine. Such objects are innovations in the system and technically do not belong to recursively or algorithmically enumerable sets on which Turing machines halt. With regard to this Mirowski (2002) has correctly asserted that mathematicians "finally have blazed the trail to a formalized logical theory of evolution "(ibid. p.141). In other words, dynamical system outcomes produced by algorithmic agents need not be computable and fail to be systematically identified by codifiable meta models. This is referred to as undecidable dynamics. Gödel's Second Incompleteness Result shows that it is precisely when systems isomorphic to number theory are consistent that internal consistency, which is a strongly self-referential system wide property often regarded as the hallmark of rational order, cannot be established by an algorithmic decision procedure. Gödel (1931) axiomatically derived the undecidable proposition, the encoding of which represents the diophantine equation which has no algorithmic solution. ${ }^{7}$ This class well known as Hilbert's Tenth problem has the highest degree of algorithmic

\footnotetext{
${ }^{7}$ Diophantine equations are polynomial equations with integer solutions. The irreducible nature of the computation here is that short of letting the system run its course there is no a priori systematic way to determine the solution to the problem.
} 
unsolvability. Penrose(1988) was amongst the first to identify so called non-computable patterns or tiling problems, that nevertheless emerge from the execution of simple rules, with the Gödel incompleteness result.

Methods of search and inductive inference in non-computable domains of problems which defy algorithmic or deductive inference has progressed substantially with methods of evolutionary/adaptive computation such as artificial neural networks (ANN) (see, Hertz et. al. 1991) and in the form of genetic algorithms (GA) and genetic programs (GP) pioneered, respectively, by John Holland (1975) and John Koza (1992). The fundamental issue with search procedures for optimal solutions is that the domain of search of all possible procedures which are fully defined, viz the class of total recursive functions, is not recursively enumerable. This set which is uncountably infinite has no algorithmic decision procedure and hence trial and error outcomes of inductive search may be far from a global optimum. Typically, algorithmically unsolvable problems fail to be categorical ${ }^{8}$ in that they have no unique decision/solution procedure. Instead, a multiplicity of heterogeneous decision rules have to be considered in parallel and distributed fashion. This is the hallmark of methods of many natural and artificial evolutionary computation such as GAs, GPs and ANNs. Holland (1975) pioneered the Darwin/Fisher principle for the selection of decision rules within the context of GAs. A decision rule is selected to grow/replicate at a rate which is in proportion to its fitness relative to the average fitness of the fixed population of decision rules. Hence, the term replicator dynamics has been given to this method of selection (see, Hofbauer and Sigmund,1998, p.67) and is thought to best suit short term evolutionary dynamics with fixed number of types in the population. Note that GAs and GPs can introduce novel decision rules by cross over and mutation operators. In general, the problem of selection when global credit assignment mechanisms on individual performance are not in place or how selective processes evolve from local interactions falls under the major theme of self-organization in CAS. The significant factors in the long run macro evolutionary trends, viz. endogenous enhancement of performance/fitness or enlarging choice domains by innovations, are increasingly being seen as intelligent strategic adaptive responses in coevolving multi species populations. Artificial life multi agent simulations pioneered at the Santa Fe Institute and elsewhere aid in a deeper understanding of the nexus between the non-anticipating nature of global outcomes and individual agent programs or rationality.

Table 1 first developed in Markose (2002) and embellished further here shows how the new evolutionary computational methods on inductive inference and emergent computation relate to the more traditional formalistic/deductive methods. What must be noted from Table 1

\footnotetext{
${ }^{8}$ At the turn of the last century, positive proof of categoricity along with completeness and consistency of formal systems, was stipulated as desirata of formal systems by the mathematician David Hilbert. Gödel (1931) overturned this as a logical impossibility.
} 
is that the axiomatically proven impossibility results on computability (Column II) delineate the classes of problems that mathematically fail to be amenable to deductive methods and why evolutionary adaptive methods have to be resorted to. Further, one may contrast the implications for Economics given at the bottom of Columns I, II and III of deducibility and incompleteness, respectively.

It is a major premise of this overview that scant attention paid by economists to the epochal non-computability issues for their science has been a stumbling block to a deeper understanding of the relevance of evolutionary methods and the principles of self-organization given in Column III, Table 1. Hayek $(1952,1967,1982)$ was one of the first economists who explicitly espoused the Gödelian formalist incompleteness and impossibility limits on calculation which he referred to as the limits of constructivist reason. This led Hayek to the necessity of evolutionary solutions with the abiding premise of his large ouvre of work being that market institutions which coevolved with human reason enable us to solve problems which are impossible to do so by direct rational calculation. Using different formulations of the problem, Lewis (1987) and Spear (1989) seminally showed the generic non-computability of fixed point mappings that represent equilibria in markets. Axtell's contribution, this issue, and pioneering work by Arifovic (1994) on evolutionary learning of rational expectations and inductive methods for equilibrium selection (see, survey in Arifovic, 2001) arise because of non-computability issues or because of real time computational intractability of fixed point mappings. Velupillai (2000) derived the diophantine complexity class of parallel distributed computing that arises in a game where cooperation and competitive innovation can arise. The non-computability of fixed points with contrarian players, the mainstay of Gödelian logic and that of the halting problem will also be shown to be pertinent to the very large area pioneered by Brian Arthur on the nature of self- organization in stock market environments. As we will see, the Binmore (1987) use of the Gödelian logic and Turing machine agents in a game is shown by Markose(2001b, 2002, Section 3) to be of fundamental importance in resolving one of mysteries surrounding CAS on the emergence of new forms. Thus, while Velupillai (2000) succintly points out how problems of non-computability naturally reside close at hand for cognitive aspects of choice, following Binmore (1987) this overview underscores theoretical reasons that market agents can be assumed to have full computational capabilities of Turing machines and why in no small measure the non-computable environment that necessitates the inductive methods of Table $\mathbf{1}$ Column III are endogenously generated by the activity of such agents. 
Table 1

\begin{tabular}{|c|c|c|}
\hline $\begin{array}{l}\text { Formalistic Methods } \\
\text { *Predicate and } \\
\text { Propositional Calculus } \\
\text { *Classical methods of } \\
\text { optimization } \\
\text { * Classical } \\
\text { Probability and } \\
\text { Econometric Models }\end{array}$ & $\begin{array}{l}\text { II.The 'New Logic': } \\
\text { Mathematics of } \\
\text { Incompleteness } \\
\text { \#Gödel(1931): } \\
\text { Self-reference, } \\
\text { Undecidability and } \\
\text { Incompleteness } \\
\text { \#Church-Turing-Post: } \\
\text { Algorithmic Unsolvability; } \\
\text { Halting Problems } \\
\text { \# Wolfram-Langton } \\
\text { Complexity class with Type } \\
\text { IV Dynamics and phase } \\
\text { transition: "life at the edge of } \\
\text { chaos". } \\
\text { Computability Methods } \\
\text { *Recursion Function/ } \\
\text { Computability Theory } \\
\text { *Algorithmic and Stochastic } \\
\text { Complexity Theory }\end{array}$ & $\begin{array}{l}\text { III.Inductive Methods and Self } \\
\text { Organizing Dynamics } \\
\text { \#Theory of Emergence } \\
\text { And Self-Organizing Complex } \\
\text { Systems (Local interaction } \\
\text { models with spatial topology of } \\
\text { networks: Models of self- } \\
\text { organization with endogenous } \\
\text { production of adaptive novelty) } \\
\text { \#Non-computable Penrose tiling } \\
\text { and pattern generation } \\
\text { \#Coevolving multi species and } \\
\text { Red Queen Effects } \\
\text { Methods of Adaptive } \\
\text { Computing } \\
\text { *Cellular Automata } \\
\text { *Classifier Systems } \\
\text { *Genetic Algorithms and } \\
\text { Genetic Programs } \\
\text { *Neural Networks } \\
\text { *Numerical multi-agent } \\
\text { simulations }\end{array}$ \\
\hline \multicolumn{3}{|c|}{ IMPLICATIONS FOR ECONOMIC MODELS } \\
\hline $\begin{array}{l}\text { Perfect competition; } \\
\text { existence results in } \\
\text { general equilibrium } \\
\text { models and static } \\
\text { Welfare analysis } \\
\text { - } \quad \text { Command Economy } \\
\text { - Eductive Game Theory } \\
\text { - } \quad \text { Evolutionary Game } \\
\text { Theory and diffusion } \\
\text { dynamics with no new } \\
\text { forms } \\
\text { - Lognormal Stock } \\
\text { Market Models } \\
\text { - Homogenous rational } \\
\text { expectations } \\
\text { Uniqueness and } \\
\text { homogeneity with } \\
\text { Type I (limit points), } \\
\text { Type II (limit cycle) } \\
\text { dynamics } \\
\text { Market Completeness }\end{array}$ & $\begin{array}{l}\text { - Hayek }(1967,1982) \text { on the } \\
\text { limits of constructivist } \\
\text { reason; Hayek } \\
\text { (1952,1982) on cognitive } \\
\text { incompleterness } \\
\text { - Lewis }(1985,1987) \text { on } \\
\text { algorithmic unsolvability } \\
\text { of general equilibrium } \\
\text { prices } \\
\text { - Spear (1989) on the generic } \\
\text { non-computability of } \\
\text { Rational Expectations fixed } \\
\text { points } \\
\text { Vellupillai ( 2002) on } \\
\text { Diophantine complexity of } \\
\text { strongly self-referential } \\
\text { global encoding of parallel } \\
\text { distributed computation } \\
\text { Binmore(1987)/Markose } \\
\text { (2001b,2002) non- } \\
\text { computability of fixed } \\
\text { points with contrarian } \\
\text { agents and strategic } \\
\text { necessity for innovation }\end{array}$ & $\begin{array}{l}\text { - Irregular innovation based } \\
\text { structure changing dynamics } \\
\text { in capitalist growth: Type IV } \\
\text { undecidable dynamics } \\
\text { - } \quad \text { Stock market crashes and non } \\
\text { Guassian asset returns with } \\
\text { fat tails } \\
\text { - Red Queen dynamics with } \\
\text { rivalrous arms race in product } \\
\text { innovation, regulatory } \\
\text { arbitrage etc. } \\
\text { - Critical heterogeneity } \\
\text { - Information/technology } \\
\text { diffusion in socio-economic } \\
\text { network with path } \\
\text { dependence, increasing } \\
\text { returns to scale } \\
\text { - Evolved norms and } \\
\text { institutions for self- } \\
\text { organization: computational } \\
\text { efficiency versus maladaptive } \\
\text { entrainment } \\
\text { Market Incompleteness }\end{array}$ \\
\hline
\end{tabular}


In the case of the choice problem, even a simple evaluation of the truth value of " $\mathrm{X}$ or else $\mathrm{Y}$ "type proposition which is necessary for categorization of objects of choice, involves calculations of the neuronal network that are non-linear, of over dimension three, along with the assignment of integer or rational valued weights as in the solution of a diophantine equation, Velupillai (2000). As no uniform solution procedures exist for this, even the perfectly rational agent with full computational powers qua Turing machine will still arrive at a solution in an adaptive way using trial and error techniques. ${ }^{9}$ As with the above cognitive problem of classification, solutions once learnt by trial and error may be retained and passed down in some hereditary process. System wide adaptive shared operational schema can emerge and these can then be called upon habitually when certain if-then conditions are satisfied. Inductive inference or behaviour of this kind which requires no de novo solutions is instinctive, atrophied into skilful behaviour within an adaptive shared schema and may in the words of Gode and Sunder (1993) display zero intelligence. Examples will be given below of this important aspect of adaptive learning of shared operational schema in self-organization especially in socio-economic systems.

\subsection{Perspectives on Self Organization}

\subsubsection{Self-organization In Systems With Endogenous Production of Novelty}

Following the von Neumann legacy that transformed the foundations of dynamics from energy and motion to information and computation, Wolfram (1984), in what is now called the Wolfram-Chomsky schema, postulates that on varying the computational capabilities of agents, different system wide dynamics can be generated (see, Wolfram, 1984, Dawid, 1999, Foley, in Albin, 1988, pp.42-55, Markose, 2001a). Finite automata produce Type I dynamics with unique limit points or homogeneity; push down automata produce Type II dynamics with limit cycles; linear bounded automata generate Type III chaotic output trajectories with strange attractors. The significance of this schema is that it postulates that only agents with the full powers of Turing machines capable of simulating other Turing machines, which Wolfram calls computational universality, can produce Type IV irregular innovation based structure changing dynamics associated with evolutionary biology and capitalist growth. ${ }^{10}$ However, what appears mysterious is why agents with the highest level of computational intelligence are necessary to produce innovative outcomes in Type IV dynamics. The legacy of von Neumann towards what Goldberg calls a "computational theory of actor innovation "(see, footnote 3) makes a veritable break with traditional Darwinian tenet that the

\footnotetext{
${ }^{9}$ In other words, the popular assumption that agents need to have some arbitrary bounds on their computational capacity to justify the adaptive methods of Table I Column II, may be a mistaken one.

${ }^{10} \mathrm{It}$ is a matter of controversy as to what conditions produce computational universal agents. While there is agreement that far fewer than the 29-state cellular automata that von Neumann first considered could lead to structures identifiable with a universal computer, there is some doubt whether this is possible in
} 
source of innovation or mutation in the system is random. Section 3 will indicate that highly commonplace oppositional structures that proliferate in society and immune systems are the clue to understanding what agents, if they were Turing machines, would do.

What is remarkable is that the formal character of equilibria of systems capable of the endogenous production of novelty based complex dynamics corresponds to the notion of recursively inseparable sets first defined by Emil Post (1944) in the context of algorithmically undecidable decision problems. It is in this context, that Casti (1994, pp. 143-149) makes the connection between complex undecidable dynamics and 'surprises'. Langton $(1990,1992)$ identifies the analog between the Wolfram-Chomsky complexity classes, the halting problem and the phenomenon of phase transitions and colourfully refers to the phase transition associated with Type IV dynamics as "life at the edge of chaos". The recursively inseparable sets are two disjoint sets. ${ }^{11}$ The one on which Turing machines halt is associated with Type I

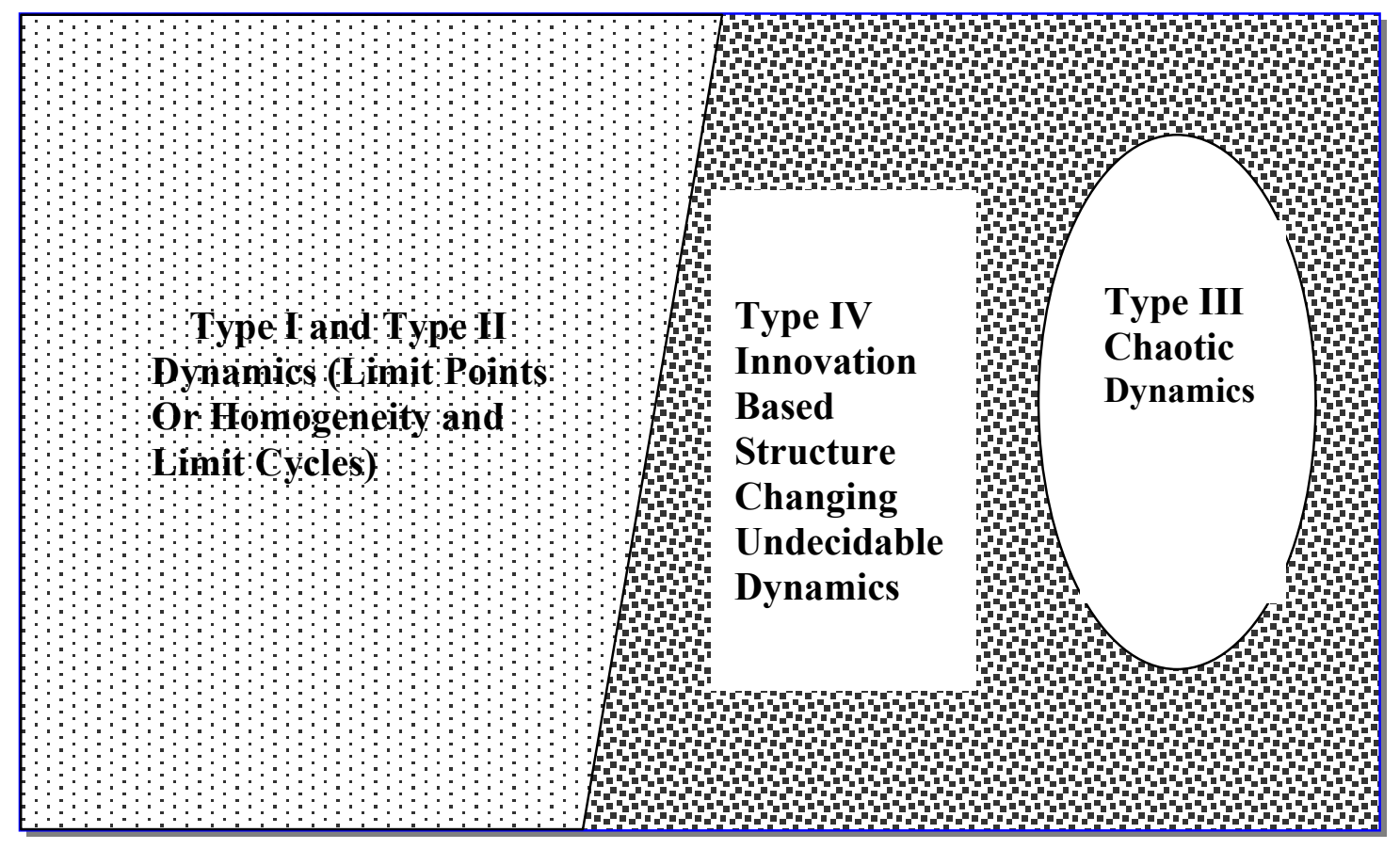

Fig.1 Set Theoretic Representation of Self-Organization With Endogenous Novelty

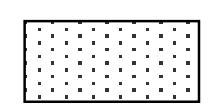

Set on

Which

Turing

Machines

halt

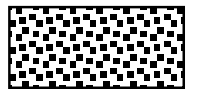

Set on

which

Turing

machines do

not halt

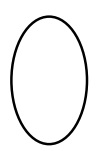

Set on which

Turing machines

can be deduced

not to halt

the 2-state cellular automata that Wolfram has considered (see, Bak, 1996, p.106-107) The Langton (1990) experiments with a 5 state cellular automata which appears to give him Type IV dynamics.

${ }^{11}$ Technically, these disjoint sets are recursively enumerable but their complements are not. If this were not to be the case, the system will be complete in that no novel encoded objects (not already in these sets) can ever arise. 
and Type II dynamical systems with limit points and limit cycles, which Langton calls (computable) order. The other set on which Turing Machines can be logically deduced to be incapable of halting represents systems with Type III chaotic dynamics. Both of these sets represent attractors for dynamical systems that cannot produce novelty. The domain for novelty producing Type IV dynamics lies outside both these sets.

Figure 1 gives the set theoretic representation of the Wolfram-Chomsky schema of the complexity classes for dynamical systems which formally corresponds to Post's set theoretic proof of the Gödel Incompleteness Result (see, Cutland, 1980, Markose, 2001b).

It is indeed the case that the necessity for self-organization of a system capable of endogenous novelty production follows from this axiomatic proof on the impossibility of its algorithmic implementation. No finite meta model can ever computably identify the novelty based change in the structure of this system. As will be seen in Section 4, this has an empirically testable hypothesis well known to economists as the Lucas Critique (Lucas,1976) on the failure of econometric models to identify structural breaks that follow from strategically induced innovation.

Langton $(1990,1992)$ makes an important observation that systems capable of endogenous novelty generation experience a critical slowing down at the phase transition between the two other domains of (computable) order and chaos as the system at this juncture is effectively involved in an irreducible process of calculation of an undecidable global ordering problem of Gödel's Diophantine degree of complexity. An important aspect of the nonrecursive reordering or self-organization that is going on when innovations have to be incorporated into an extant system is that the logical consistency of the internal rules/programs governing the dynamical system must be satisfied at the phase transition. The big question addressed in the next section is whether Langton's self- organized phase transition associated with Type IV complex dynamics can arise in systems that are incapable of endogenous novelty production and where innovations are exogenously introduced.

\subsubsection{On Self-Organized Criticality (SOC) With Exogenous Innovations: Connectivity and} Networks

Bak and his coworkers (Bak et. al. ( 1987), Bak and Sneppen (1997)) have been very influential in associating some universal macroscopic properties, in particular, the statistical distribution of power laws in systems with large ensembles of micro interacting constituent parts. Power laws have been observed in natural, artificial and social systems ranging from earth quakes, the size of cities and to income distribution. The discovery by Pareto (1887) of 
the power law exponent of $3 / 2$ for wealth distribution ${ }^{12}$ which has stayed remarkably constant over two centuries for capitalist countries, is perhaps the most famous of such laws.

Bak postulates that power laws are the statistical consequence of systems that are capable of self-organization at critical points. Bak (1997, p. 112) clearly would like to identify his principle of self-organized criticality precisely with systems that have computational universality, viz. those capable of endogenous production of novelty. In this framework, a sub critical system will converge to homogeneity and a supercritical system will manifest chaotic dynamics with critical behaviour lying between the two as laid out in Figure 1. Despite, the precise mathematical characterization of Langton's "life at the edge of chaos" as the critical phase transition associated with Type IV complex dynamics, the latter has unfortunately been the subject of much pastiche, invoked even in the case of systems without endogenous capabilities for generating novelty.

The nature of dynamics associated with self-organization is qualitatively well known as irregular 'waves' or avalanches' observable from the process of spontaneous reorganization in capitalist creative destruction and also from records of mass extinctions in evolutionary biology. The Bak hypothesis is that in SOC systems, the number of individuals involved in each wave of birth/death type reorganization that follows from a 'mutation' in the system will follow a power law. This power law exponent should also be invariant across different scales of population size when the system is critical.

The deficiency, however, with the computer simulation models that Bak and his coworkers have devised, the so called sandpile model and the Bak- Sneppen (1993) model on waves of evolutionary extinctions, is that the systems per se do not have any capacity to produce innovation endogenously. In the simple version of the Bak-Sneppen (1993) model with species arranged on a one dimensional lattice, the least fit species $\left(f_{\min }\right)$, where fitness is a number between 0 and 1, is exogenously subjected to a random mutation and so are the two adjoining species with their new fitness levels being drawn from a uniform distribution. This fixed dynamical rule when iterated a large number of times produces burst like avalanches. A critical value, $f_{c}$, in the limit is identified for the level of fitness in the population below which the probability distribution of fitness falls to zero and above it the distribution converges to a fixed value. ${ }^{13}$

\footnotetext{
${ }^{12}$ The probability distribution (or the proportion of individuals in a population with wealth of size w) is given as $\mathrm{P}(\mathrm{w}) \sim \mathrm{w}^{-1-\alpha}$. Here, $\alpha \sim 3 / 2$ is what is referred to as the power law exponent. See, also Durlauf, this issue, for more economic examples.

${ }^{13}$ For any given level of fitness $0<\mathrm{f}^{*}<1$, an avalanche of duration $\mathrm{T}$ is defined as follows: for $\mathrm{t}<\mathrm{t}^{\prime}<\mathrm{T}$, $f_{\min }(t)>f^{*}, f_{\min }\left(t^{\prime}\right)<f^{*}, f_{\min }(T+1)>f^{*}$. That is, the duration of the avalanche is the time $T$ for which the species with the minimum fitness $f_{\min }$ in the population starting from the point at which it exceeds the given level of fitness $f^{*}$, falls below it and then recovers again. Bak and Sneppen(1993) find that for $f_{c}$,
} 
The Ising model in statistical mechanics adopted and extended by Liggett $(1985,1997)$ is the fundamental prototype for the dynamics arising from large numbers of local interactions. However, Ligett $(1985,1997)$ makes no claims for self-organized criticality, Type IV dynamics, or for powers laws. Further, for reasons that will be obvious, it will be useful to refer to Ligett criticality (to distinguish it from Bak SOC) as the point at which systems tip in one direction or another. Typically, in the Ligett/Ising framework the diffusion of a binary process is considered, such as infection or no infection in epidemics, to buy or sell in a herding model pertaining to markets, choice between two competing products etc. It can be argued that in the absence of endogenous scope for novelty, there is little to mathematically differentiate between Bak criticality and Ligett criticality except the focus of analysis. Thus, notwithstanding the controversy on whether the agency of change can be assumed to be exogenous, as has been expertly summarized in Jensen (1998, p.126-127), the two necessary and if not sufficient conditions in such spontaneous reorganization with critical power law behaviour is interconnectivity in local rules of agents and the application of thresholds. Further, some systems with large numbers of interacting elements may also have network effects which form positive non-linear feed back loops resulting in increasing returns to scale.

The crucial insight here is on the connectivity of agents in networks and that criticality in systems may also be viewed as those displaying specific classes of patterns of connectivity or networks. In graph theory nodes/vertices can be represented as agents and edges as connective links with a function (fixed or time varying) measuring the intensity or frequency of interaction between $(\mathrm{i}, \mathrm{j})$ agents. ${ }^{14}$ On the one extreme there are networks where every player can interact with any other (a zero dimensional one) or one where a player can interact with its nearest $(\mathrm{k})$ neighbours. The small world networks (Watts and Strogatz (1998), Watts (2002)) which are meant to be characteristic of socio economic networks, is one in which the fixed kneighbourhood of an individual is modified by breaking a fraction of its $\mathrm{k}$ original links. An equal number of new links are created by adding to an individual's neighbourhood a set of randomly selected individuals from the whole system.

As with horses for courses, economic applications of local interacting agent models have taken two types of perspectives. The first perspective favoured by econo physicists and some ACEs has gone down the Bak route with the focus on self organized criticality and power laws. Here, the Lux and Marchesi (1999) stock market model with local level fixed nonadaptive interactions producing fat tail global price dynamics has been influential. In a recent application of the Bak-Sneppen type framework for the study of technological diffusion, Arenas

the probability density function of the so called avalanches of different durations, has a power law distribution, $\mathrm{P}(\mathrm{T}) \sim 1 / \mathrm{T}$.

${ }^{14}$ Mathematically, Green (1994) has found that the many different representations of interconnectivities in systems such as in matrix models (for example, Markov processes), dynamical systems involving differential equations and cellular automata are all isomorphic to directed graphs. 
et. al. (2002) consider changes driven by cumulative social interactions spreading through social networks with the threshold cost of adoption being dependent on the degree of incompatibility with neighbours. Agents are placed on a one dimensional lattice and an exogenous selection is made of agent to have his technological competence enhanced by a random amount in periods when diffusion waves have died out. Arenas et. al. (2002) define a parameter k which reflects the relative incompatibility costs of being either ahead of one's neighbours or behind them. Each agent plays a myopic best response as in a coordination game with the important proviso that agent i's game with $i-1$ is not independent of what he plays with $i+1$. They find that below a critical value for $\mathrm{k}$, denoted by $\mathrm{k}^{*}$, the system shows complete homogeneity in technological competence used and at $\mathrm{k}^{*}$ a critical degree of heterogeneity persists with power laws observed in the size of avalanches. An avalanche is defined by the number of agents who revise their technological level upwards after a random update on the technology of one agent. It must be noted that Arenas et. al (2002) do not show self-organization with $\mathrm{k}^{*}$ being obtained by an endogenous process. They give a series of simulations of the system dynamics with different values of $\mathrm{k}$ and report the critical $\mathrm{k}^{*}$ as one for which the size of avalanches follows a power law with an identical exponent irrespective of scale of the population. This is not satisfactory along with the pastiche on Langton's characterization of Type IV dynamics for their system with no endogenous capabilities for innovation. However, what is important to note is that the critical heterogeneity in adoption strategies that arises from the power law characterization of self-organizing systems is in sharp contrast with the bulk of extant evolutionary game theory models on coordination games. Convergence to homogeneity of strategies in the latter appears to be the norm in contrast to the rich diversity observed in the real world.

A very large group of local interaction economic models (see, Durlauf and Young, 2000) that comes under the rubric of social dynamics for the study of the diffusion of innovation, information or norms is based on the Liggett/Ising framework adapted for economic analysis by Blume (1993). This framework treats local feedback effects as a stochastic process in which the probability that a given person adopts one of two possible actions, say $\mathbf{A}$ or $\mathbf{B}$ in a given period of time, is assumed to be an increasing function of the number of his neighbours who have adopted it. ${ }^{15}$ There is also an idiosyncratic factor that reflects agents' preference for $\mathbf{A}$ or $\mathbf{B}$ irrespective of other agents. Many of these models assume a fixed network structure

\footnotetext{
${ }^{15}$ A well known formulation of this in the stock market context, Sornette (2003), is for an investor, i, to do what the majority of his neighbours did in the last period (sign assumes ' + ' for majority buys and '-' for majority sells) up to an idiosyncratic noise term $\varepsilon$. Thus, $\mathrm{s}_{\mathrm{i}}=\operatorname{sign}\left(\mathrm{K} \sum_{\mathrm{j} \in \mathrm{N}_{\mathrm{i}}} \mathrm{s}_{\mathrm{jt}-1}+\varepsilon\right)$. The factor

$\mathrm{K}$ is inversely related to market depth or the trading volume and $\mathrm{N}_{\mathrm{i}}$ are those investors directly connected to the ith. investor. The relative strengths of the cohesion/imitative parameter $\mathrm{K}$ and the variance of idiosyncratic term with a critical value for $\mathrm{K}$, defined as $\mathrm{K}_{\mathrm{c}}$, determines whether one way markets form or whether the idiosyncratic noise impedes this. In the critical state there are equal numbers of buys and
} 
though endogenously changing networks have been considered in Mailath et. al. (1997) and Jackson and Watts (2002). The impact of the spatial interconnectedness or the network structure on the diffusion dynamics has been analysed on a case by case basis by Goyal and Janssen (1996), Chwe (2000) and Morris (2000). The state of the system at each time period is the vector of A's and B's adopted by each of the agents who are represented by vertices of the graph defining the social network. The potential of a state is the linear combination of the payoffs from the A-region, the B- region and the idiosyncratic component. Following the Ising model and statistical mechanics, the long run relative frequency of each state is given by the Gibbs distribution which is concentrated on the states with high potential. Such states have been defined to be stochastically stable by Foster and Young (1990).

As already noted, in contrast to the Bak type criticality literature which studies the critical state of the system when both $\mathbf{A}$ and $\mathbf{B}$ coexist, the tipping style Ising models of socioeconomic dynamics have focused on asymptotic stochastic stability analysis. The bulk of what passes as complex systems theory currently is the local interaction models of systems that are not capable of producing innovation or ones in which innovations are exogenously introduced. The network dynamics for 2-dimensional Ising model and some of its hierarchical extensions are fully solvable with many of the economic applications here being fully analytical. However, it is far from the case that in the models surveyed here critical behaviour is self-organized. It is increasingly being understood that the self-organization in a system requires endogenizing the scope for competitive coevolutionary dynamics referred to as the Red Queen principle which maintains the system at the critical region. This will be addressed in Section 3 .

\subsubsection{Self-Organization by evolution of common shared operating schema or institutions}

This section grapples with the idea that on the one hand while cooperative behaviour is a strong force of self-organization, on the other, as seminally mooted by Axelrod $(1984,1987)$ cooperative behaviour and such societal norms may be an emergent phenomenon rather than arising from rational calculation. It is well known that the two person one shot version of the Prisoners' Dilemma ${ }^{16}$ leads to a Nash equilibrium outcome that favours the dominant strategy to defect rather than to cooperate. In what Axelrod calls iterated Prisoners' Dilemma (IPD), players remember others they previously interact with and also some aspects of the prior outcomes. Axelrod invited a number of game theorists to submit strategies that they believe would win the highest scores when playing one another, a pair at a time, in a round robin

sells, however, there are large clusters of consensual behaviour with the system being highly susceptible to unstable crash type behaviour.

${ }^{16}$ Purists on emergent outcomes would argue that cooperative behaviour at a collective level should be shown to arise from agents who have no inherent mechanism for cooperation embodied in them. In other words, what the observer would categorize as cooperative behaviour at a collective level should not be in 
tournament. So, Computational Experimental Economics was born. On the face of it, given entrenched priors on what one thinks is rational play, the Bayesian strategy with the other player's moves modelled as a Markov process (one of the strategies that was submitted) could be thought to be the winner. Interestingly, the simplest strategy called Tit-For-Tat submitted by Anatol Rapoport achieved the highest average score. In a series of computer simulations of IPD where agents learn to play robust responses using genetic algorithms in repeated rounds ${ }^{17}$, a whole new notion of robustness of rules in coevolving populations of strategies was mooted by Axelrod. Recently, N person IPD has been generalized to include spatial topology in the interaction of agents, especially in a small-world framework. In Abramson and Kuperson (2001) it was found that when the connective properties were altered from a completely ordered lattice to a random network, for fixed payoffs in the game of N- person IPD, the emergent behaviours that it sustained were non-trivially different.

The classic example of the emergence of a common shared operational scheme that then coordinates large numbers of decentralized trading agents in economic systems is the emergence of money as a medium of exchange. This is no different from the coordination achieved in slime mold composed of constituent cells with none being dictatorial in any way but each having the capacity to respond to a common chemical signal, Keller and Segel (1970). This enables the slime mold to self-organize as if as one and 'walk' across the forest floor in large clusters. The 'walking' is done as a single unit as there is a positive feedback from the larger quantities of chemicals emitted from clusters to individuals encouraging the growth of larger clusters.

Carl Menger (1892) had conjectured that a medium of exchange could have emerged spontaneously rather then by dictates of the sovereign. Marimon et. al. (1989) consider a trading regime where there is an absence of conditions that permit barter, viz. the double coincidence of wants. Further, in addition to goods that agents produce and consume (each type of agent exclusively produces one good that he does not consume and consumes a strict subset of others) there is one good that they neither produce or consume, say good 0 . The experiment uses artificially intelligent agents who meet randomly and make decisions to trade, consume or carry forward goods which are acquired only in the expectation that it may lead to more frequent trades in the goods that the agent consumes. Agents use two Classifier Systems with a scoring/credit accounting system to select rules for trading and another one for consumption

the local objective/payoff function at the level of the individual. An example of this is given in Hemelrijk (1997) where herd behaviour emerges in aggressive individuals.

${ }^{17}$ Based on evidence in Linster (1992), Binmore (1998) has criticized Axelrod's claim that Tit-For-Tat or strategies 'similar' to it dominated the runs in which cooperation emerged in the IPD version with agents selecting strategies using genetic algorithms. Making the notion of strategies that resemble Tit-For-Tat in machine code more precise, Tsang et. al (2003) show that though the main Axelrod premise that the majority of the converged runs shows that cooperation emerges, Tit-For-Tat like strategies only account for $20.1 \%$ of all strategies and Grim comes in ahead of all strategies at $24.9 \%$. 
decisions. Agents can improve welfare by coordinating on the use of good 0 as a medium of exchange. But, there is no a priori way of deducing which trading/consumption rules when mutually adopted will lead to this. In the Marimon et. al. (1989) model, over time it was discovered that the artificially intelligent agents with minimal decentralized information and aiming only that they increase their utility, inductively (Column III, Table 1) found rules that led to good 0 being the common medium of exchange.

One of the important insights from multi-agent adaptive simulations such as by Ackley and Littman (1992) is the inverse relationship between the need for explicit calculation and learning and the evolutionary development of system wide well adapted operational schema or institutions. Once the latter are in place agents need to exercise very little intelligence to get things 'right'. What explicit learning was initially needed when fully adapted becomes instinct and atrophied into skilled behaviour. In the Ackley and Litterman (1992) experiments, agents have neural network brains which are capable of being universal function approximators with the same powers of calculation as Turing Machines. The apparent bounded behaviour in brain activity that is seen after the emergence of shared schema should not therefore lead one to conclude that the agents have any ad hoc bound to their computational intelligence in the first place.

The recent experimental behavioural studies surrounding the variants of double auctions show that the market in action can produce easy convergence to competitive market clearing prices with very few traders who use a minimum of strategic behaviour or computational powers. In a now celebrated paper, Gode and Sunder (1993) show that continuous double auction markets populated by zero intelligent agents are highly efficient in extracting gains from trade and price trajectories converge to competitive equilibrium prices. Zero intelligence corresponds to simple computer programs that generate random bids (or asks) subject to a no loss constraint. The latter means that traders cannot buy above their redemption values or sell below their costs and no attempt is made to maximize profits. This was sufficient to obtain $98 \%$ of the gains from trade. Thus, the best price rule of execution in double auction ${ }^{18}$ is a simple but powerful device to obtain competitive outcomes with the great economies of computation and information that Hayek (1945) emphasized. Sunder (2002) concludes that "a claim that the predictions of the first fundamental theorem in economics are approachable in classical environments without actual or attempted maximization by participants might have been met with scepticism until recently. Thanks to a largely serendipitous discovery using computer simulations of markets, we claim that weak forms for individual rationality, far short of

\footnotetext{
${ }^{18}$ In a double auction a buyer submits a price at which he is willing to buy ( bid) and the seller submits the price at which he will sell (ask). The rule of best price execution states that at any point in time execution of trades occurs at the highest bid in the market and the lowest ask. Those buyers and sellers who cannot meet this are put under competitive pressure.
} 
maximization, when combined with appropriate market institutions, can be sufficient for the market outcomes to approach the predictions of the first welfare theorem" ( italics added).

\section{The Computation and Identification of Fixed Point Mappings of Market Equilibria}

2.1The computational complexity of Brouwer's fixed point and decentralized exchange Axtell's important contribution to this Feature on the prevalence of decentralized exchange is to show that it overcomes the problem of computational intractability of the Brouwer's fixed point theorem that underpins Walrasian equilibrium models. This is in keeping with the evolutionary solutions discussed in the previous section that enable us to solve problems that may almost surely be beyond methods given in Column I, Table 1. A problem is computationally intractable if it only has exponential time algorithms that have computation times that vary exponentially with the problem size (an integer $\mathrm{N}$ ), for example, $\mathrm{r}^{\mathrm{N}}$, for some $\mathrm{r}$ $>1$. A polynomial (P) time algorithm is said to be tractable if its computation time varies proportional to the problem size raised to some integer power, $\mathrm{d}$, as in $\mathrm{N}^{\mathrm{d}}$. Exponential functions grow strictly faster than polynomial ones. The significance of P-class problems is that they coincide with those that can be realistically solved by computers and it is held that every practical and efficient algorithm can be rendered as a polynomial time bounded Turing machine.

In general equilibrium theory of markets ( Arrow and Hahn, 1971) it was thought that the burden of proof placed by the invisible hand type argument involved the establishment of a formal possibility or an existence result on an equilibrium in a decentralized economy. Here individuals motivated by self interest and guided by price signals alone are postulated to enjoy consistency of economic plans and Pareto optimal resource allocation. The fixed point theorems of Brouwer and Kakutani that imply generic existence results for equilibrium prices is the mathematical tool that gives credence to the Walrasian model of an exchange economy.

Mathematical problems where a solution is known to exist as in the case of the existence results of Kakutani and Brouwer are called function problems(FP). This is in contrast to the structure of decision problems where a yes or no answer is sought to whether the problem has a solution. Typically, for function problems more elaborate output than "yes" or "no" is required. Axtell, this issue, uses recent results on the complexity class of Brouwer type function problems for fixed points (see, Papadimitrou ,1994) which show them to be related to that of the parity argument via the Sperner's Lemma. ${ }^{19}$ The parity argument arises from a graph theoretic lemma that any finite graph has an even number of odd-degree nodes. Sperner's Lemma guarantees the existence of a directed graph to the 3-colored triangle identical to the larger one, but to date no known polynomial algorithm exists for this. The analogue to

\footnotetext{
${ }^{19}$ Sperner's lemma states that any admissible colouring of any triangulation of the unit triangle has a 3coloured triangle, (viz. an odd number of them by the parity argument.). While a directed graph to the 3colored triangle (within the larger triangle) exists, the constructive problem is that there is as yet no known polynomial time algorithm for this.
} 
Brouwer's fixed point theorem follows from the generalization of Sperner's Lemma when applied to a N-dimensional simplex guarantees the existence of a panchromatic simplex. The Brouwer's fixed point function $\mathrm{f}\left(\mathrm{x}^{*}\right)=\mathrm{x}^{*}$ can be proved to exist at the limit of the center, $\mathrm{x}^{*}$, of panchromatic simplices obtained by finer and finer triangulations, Papadimitrou (1994). Once again, due to the above analogue to Sperner's Lemma, there are no known polynomial algorithms for the Brouwer and Kakutani fixed points specifically in the general case of nonlinear utility functions for the Walrasian equilibrium model. Axtell concludes that the exponential (non-polynomial) complexity of the Walrasian fixed point algorithms as opposed to polynomial computations involving $\mathrm{N}$, the number of commodities in the economy, may rule them out as a plausible computational device for equilibrium price formation in real world markets.

Axtell conjectures that polynomial complexity that is a prerequisite of real world equilibrium price formation mechanisms may crucially arise from institutional arrangements that promote informational and computational decentralization. The aggregation of information into the net demand functions of the Walrasian model has long been known to be excessive. The much quoted observation regarding this being that of Hayek (1945, italics added) "We cannot expect that this problem will be solved by first communicating all this information to a central board which, after integrating all knowledge, issues its orders ... The problem is to show how a solution is produced by interaction of people each of whom has partial knowledge".

However, it is not till very recently that it has been recognized that the invisible hand type arguments also involves a burden of proof along the lines of what John Rust (1987) has called computational decentralization that militates against the highly aggregative calculation involved in the fixed point algorithm of the Walrasian model.

Axtell, this issue, suggests that instead of imputing the locus of equilibrium price calculations to reside in an entity such as the Walrasian auctioneer, it is more appropriate to consider the decentralized market as a whole as a collective computing device. Axtell then proceeds to show that parallel distributed agent based models of k-lateral exchange can restore the all important polynomial complexity in calculation of equilibrium prices and allocations.

\subsection{Adaptive/Inductive Learning of Rational Expectations Equilibria}

A major part of the coordination processes in markets rely on the identification and calculation of fixed point mappings of global signals such as equilibrium prices. By forming rational expectations of fixed points of market equilibrium prices economic agents are meant to coordinate their activity and iron out inconsistent expectations. Spear (1989) was the first to show that the problem of identifying a set of fixed points for market equilibrium price functions 
is algorithmically unsolvable. ${ }^{20}$ The absence of an unique decision procedure or a deductive means by which to select appropriate forecast models/functions has been intuitively justified as arising from the self-referential character of the problem by Arthur et. al. (1997) : "the expectational models investors choose affect the price sequence, so that ..their.. very choices of models affect their data and so their choices of model. Arthur et. al. (1997) refer to the above self- referential structure of the problem as causing it to be ill defined. Likewise, in the El Farol game, Arthur (1994) gives the classic prototype of a problem with a contrarian structure for which there is no deductive means of arriving at a solution. The methods of Table 1 Column II can precisely show how these problems are well understood to be algorithmically unsolvable. Mathematically to solve such problems, methods of inductive inference including that of heterogeneous adaptive agents of Column III,Table 1 are required.

Arifovic (1994) pioneered the first adaptive social learning model of a homogenous rational expectations equilibrium using a single population genetic algorithm. However, in the El Farol game of Arthur (1994) where punters at a popular bar want like to be there on days when it is not crowded, the very notion of homogenous rational expectations is inappropriate to the problem. The El Farol game presents a commonplace preference to be in the minority or a situation when pay offs are greatest whilst being contrarian. An example of this is to be a buyer (seller) when the majority is selling (buying). It is intuitively clear that if all agents share the same forecasting model then actions taken by agents which are consistent with the forecast will defeat their objective to be in the minority. The fixed point of such a game involving a contrarian structure represents a non-computable fixed point, see Markose ( 2001b, 2002, Section 2). In the absence of computable fixed points, rational agents even with the same information must agree to disagree. Indeed, in the large literature that has since grown on the Minority game (see, Challet and Zhang ,1998) the self-organized equilibrium that arises in the absence of computable homogenous rational expectations is sustained by a critical degree of heterogeneity in strategies. This self organized equilibrium attains greater Pareto improving welfare gains than the Nash equilibrium outcome in which agents randomize. ${ }^{21}$

In the Arthur et. al. (1997) agent based stock market model all the standard neoclassical features with preferences, constraints and even optimal portfolio choice rules

\footnotetext{
${ }^{20}$ In the computability framework, rational expectations involves an executable meta forecast function whose code represents a fixed point of the market clearing price function. This has been precisely stated in Markose (2002, Section 2).

${ }^{21}$ Denoting all $\mathrm{N}$ traders as buyers, $\mathrm{N}_{\mathrm{b}}$, or sellers, $\mathrm{N}_{\mathrm{s}}$, the 'social' gains from trade are optimized when after some large number of trading periods, $\mathrm{T}$, the following variance function $\sigma^{2}=$

$\frac{1}{\mathrm{~T}} \sum_{\mathrm{t}=1}^{\mathrm{T}}\left(\mathrm{N}_{\mathrm{bt}}-\frac{\mathrm{N}}{2}\right)^{2}$ is at a minimum. What is interesting is that the mixed strategy Nash equilibrium where agents randomly buy or sell with probability half is not in fact Pareto optimal or the emergent outcome. There is a generic degree of heterogeneity of strategies for any $\mathrm{N}$ for which the $\sigma^{2}$ function is minimized as the agents inductively solve the problem. In the latter, the minima for $\sigma^{2}$ are below what is obtained by the Nash equilibrium random mixed strategies.
} 
appear. The agents are however heterogeneous in their forecast models for asset returns and have to use adaptive GAs/GPs of Table 1, Column III as deductive means do not exist to solve such strongly self-referential mappings. The objectives of the agent based stock market experiment were to study the conditions under which homogenous rational expectations of stock returns follow and whether the stylized facts of fat tails and volatility clustering can be reproduced. As the market environment is maintained relatively stationary with a low intensity of search by GAs or GPs for better trading strategies, the unique homogenous rational expectations result followed in Arthur et. al. (1997) accompanied by a cessation of trade as per the no trade theorem. In contrast, when the rate of GA exploration for 'better' predictors was speeded up, the stock market prices began to show some of the historically observed properties of volatility clustering and crashes. However, as will be pointed out in the next section, in so far as the rate of performance enhancement, the timing and extent of retraining of GA/GP agents were exogenously altered by the experimenter rather than endogenously determined in terms of the Red Queen dynamic, it is difficult to talk about (see, Chen and Yeh, 2001) the selforganized or emergent nature of market efficiency.

\section{Evolution of Complexity And The Red Queen Principle}

3.1 The more things change, the more they stay the same

The Red Queen principle has been espoused by both evolutionary biologists, van Valen (1973) and physicists, Anderson (1977) to characterize a relentless arms race type dynamic of change among competing elements at a micro level with remarkable macro level stability of the system along some dimensions. Conveying these two aspects of the system dynamic, Anderson in his Nobel Prize lecture (1977) said "it is remarkable that in almost all cases interactions play a vital role, yet many results are not changed too seriously by them. ..We have gone to extraordinary lengths to make our magnetic moments..or electrons.. to stay in one place (italics added)... This situation was foreshadowed by an eminent $19^{\text {th }}$. century mathematician named Dodgson," (Ibid p.397). The latter also known as Lewis Carol has the following passage in his book Alice Through the Looking Glass which has lend itself to the so called Red Queen principle : "Well in our country " said Alice, still panting a little "you'd generally get to somewhere else if you ran very fast for a long time as we been doing." "A slow kind of country!" said the Red Queen. "Now here, you see, it takes all the running you can do, to be in the same place".

In Physics, the Red Queen principle asserts that a continuous application of offsetting or contravening force at a micro level can maintain global stability. In evolutionary biology, the rivalrous coevolution of species is seen to be a spur to the evolution of complexity itself, manifesting, however, no net gains in relative fitness. Here, we will be guided by Arthur Robson who is among the first to have discussed the Red Queen principle and its relevance for Economics. Robson represents an economist who has taken serious time out to study the 
evidence for competitive coevolution in biology to see what implications it may have for the growth of complexity in socio-economic institutions and market environments. He is of the view that the Red Queen type competition among species drives the most remarkable of macro evolutionary phenomena, viz. the emergence of innovative forms and more complex organisms. Analogous to this in the economic domain is the Schumpeterian model of capitalist growth which is characterized by monopolistic firms which develop innovative products involving new technology as barriers to entry for competing firms. The static world of Welfare Theorems of perfect competition with fixed preferences and technology - which many will accept as being relevant for local optima - cannot adequately deal with macro evolutionary trends that account for movements to global optima and the production of new and more complex forms. Such dynamic competition, which Robson thinks is bound to be more important than the precepts of static efficiency, still remains outside the ambit of much of standard economic theory.

As the Red Queen principle is a metaphor for the various ways in which competitive coevolution proceeds, it is conceivable that a full assessment of this principle to account for the rampant complexity of many biological and natural systems may remain less than perfect. In the light of vast evidence from evolutionary systems of the growth of complexity, Robson in his masterful survey, this issue, concludes that it is striking that much of extant game theory and economic literature in general overlooks the Red Queen principle and appears to labour under an erroneous view that there is no such arms race in complexity. There is not only little evidence that evolutionary systems select against complexity, as we will see, there are compelling reasons why an arms race in complexity is inevitable among intelligent agents.

In the Red Queen effects associated with the notion of coevolving populations of species, the furious pace of "running" relates to each species attempting to enhance their fitness relative to others. In the competition that governs the fight for scarce resources or in cases of direct confrontation with zero sum payoffs such as in parasite-host or predator-prey situations what matters is relative rather than absolute performance capabilities of the individuals. Certain attributes of individuals have to be enhanced relative to the same in others to maintain status quo, let alone to get ahead of the game. As with one, others are like wise involved in performance enhancement: triggering off an arms race. Since only hypothesis and conjectures but not direct tests of the Red Queen principle can be applied to evolutionary biology, Artificial Life simulations have become the means to understand the system dynamics and the distinctive features of competitive coevolution. The classic work of Ray's Tierra (1990) and Sim's creatures (1994) are based on the principle of competing coevolving species. In Hillis (1990) parasites were deliberately introduced and it was noted that competition among coevolving species could potentially prevent stagnation at local optima. Robson, this issue, gives an analytical example of how this might be the case. The evolution of intelligence itself is 
hypothesized to arise as an arms race giving rise to Machiavellian behaviour in social interactions within the same species, Robson (2002).

Robson, this issue, also gives a detailed account of how heterosexual sex which is instrumental in giving ample variation to genetic material of the species can be interpreted as a Red Queen type response to evade parasitic behaviour of microorganisms that can themselves mutate and resist antigens produced by host species.

\subsection{Some Economic Examples of Red Queen Effects}

What significance might the Red Queen principle have for Economics?

The Red Queen effect can manifest in the arms race of product innovation among firms in capitalist systems, simply for them to maintain status quo in market shares. In recent extensive work on a cross section of industries done by John Sutton, he finds strong evidence for this form

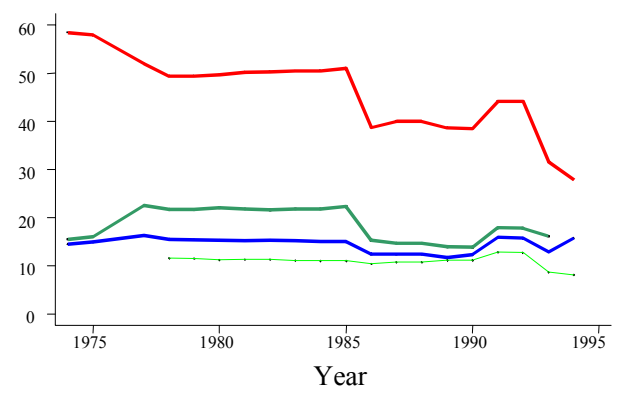

Fig2a

Market Shares :

Margarine

of non-price competition. In the case of a number industries or product categories where the leading firms were locked in a prolonged arms race of product innovation, market shares were remarkably stable, viz. rival firms' efforts offset each other. Innovating firm's products are either imitated by the rival firms or countered by their own innovations. However, there are also examples of rivalrous product innovation where the brand leaders are over taken and market shares are volatile. Failure to respond quickly enough to the competition is held up as the reason for the erosion of market share. Figure 2 a gives evidence from the margarine industry where though scores of new varieties of margarine differing in texture, flavour and hardness were introduced by the firms, their market shares remain more or less unchanged. ${ }^{22}$ In the case of cash registers, Figure 2b, where arguably the scope for technological change and

\footnotetext{
${ }^{22}$ I'm grateful to John Sutton for letting me use his findings and graphs here.
} 


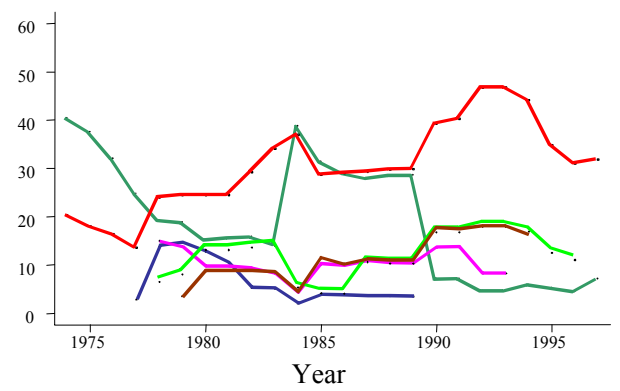

Fig2 b

Market

Shares:Cash

Registers

product innovation is vastly greater than for margarine, the market shares were highly volatile. For our purposes here either outcome is in keeping with the Red Queen principle in that players cannot maintain status quo along a critical dimension unless they can keep up with their peers. The interesting question is what global properties a system would exhibit if agents in it were relentlessly trying to 'keep up' Red Queen style.

In financial markets, enormous investment in innovative investment strategies is made to keep up and surpass the average performance of the market as a whole. In Markose et. al.(2003) the so called Red Queen constraint is endogenously imposed on the forecast and trading performance of a multi population GP agent stock market environment. The premise here is that when all speculative investors are constrained to 'beat' the market, there comes a point of market equilibrium when it is impossible for any one to do so. Market efficiency is an emergent or unintended consequence with the power law properties of investment income distribution and stock prices that satisfy the stylized facts of fat tails in asset returns.

Red Queen effects also appear in the arena of regulatory arbitrage resulting in arms race in regulatory tightening and creative evasion by regulatees. A plethora of recent institutions (see, Miller,1986) have arisen when regulatees who find it profitable to break regulatory structures do so by exiting and innovating new institutions that place them beyond the long arm of the law. The notion that surprises or innovations should be used strategically as best response because anticipated policy may be contravened by innovative regulatees has a long tradition in macro economics literature, Lucas (1972). The next section will briefly discuss the significance of Lucas's seminal insights on why the structure of opposition such as between regulators and regulatees with agents capable of anticipating the outcomes of others' strategies generates innovative structure changing Type IV dynamics.

\subsection{The ubiquitous structure of opposition and strategic innovation}


Innovation is clearly war by other means in market systems, evolutionary biology and immunology which involve oppositional zero sum parasite-host type rivalrous relationships. Ho and Saunders (1986) and more recently Kauffman (1993) have criticized the inadequacy of the Darwinian view that new forms are but a conglomeration of random variations accumulated by natural selection and preserved by heredity. In extant game theory whether eductive or evolutionary there is no notion of innovation being a Nash equilibrium strategy let alone one that is necessitated as a best response by a structure of opposition. As in traditional Darwinian evolution, in economic models innovation is either introduced at a random rate or as an ad hoc addition in the form of trend growth.

The missing piece has been the dynamic behind the emergence of variety in new forms. The Red Queen type rivalrous coevolution accounts for a contextual and strategic explanation for species being finely honed to the characteristics of other rival species. Recent empirical research on RNA virus which are considered to be the most important group of intracellular parasites has led researchers to hypothesize the notions of hyper-mutability and that of a 'mutator phenotype'. The latter is a behaviour whereby high rates of mutation are adopted with the RNA virus maintaining a highly heterogeneous population called a quasi species which generate new escape mutants that can avoid detection from the immune system. In other words, mutation rates are strategically induced rather than arising randomly and this endogenously determined dynamic sustains self-organization in the critical region. ${ }^{23}$

The Red Queen principle on having to run faster and faster to survive in the face of rivals, therefore, offers two outward manifestations of an arms race :

(1)In performance capabilities which can come in the form of enhancement of some existing faculty in the species such as intelligence, sensory faculties, speed of locomotion etc.

and/or

(2) In the use of novelty or surprises viz. the explicit production of new forms.

The ubiquitous structure of opposition envisaged in the Red Queen alone cannot suffice for the emergence of novelty or new features. As noted previously, it has long been conjectured by Wolfram (1984) that it takes agents with the highest powers of computational intelligence that resides in Turing machines and enables them to make simulations of computations made by other agents to produce innovation based structure changing dynamics. Wolfram has conducted extensive computer simulations to test his major hypothesis that computational universality is more generic than hitherto assumed and can arise in simple cellular automata (see, footnote 9). Bio-chemical or some other means by which agents can making self referential calculations of hostile behaviour of other agents is the trigger for strategic innovation envisaged in these

\footnotetext{
${ }^{23}$ The RNA virus is able to maintain a critical level of heterogeneity in the quasi species with their mutation rates being possible up to the point at which genetic coherence and heredity break down, Sole'
} 
contexts are a far cry from random mutations that have so far been held to be the only means for the emergence of new forms or 'objects'. As yet, it is not fully understood how the immune system biochemically detects hostile agents (see, Holland, 1992). Markose (2001b, 2002, Section 3) uses the epochal Gödel (1931) incompleteness result to show that the conditions of opposition between two Turing machines and for these machines to mutually recognize this structure are logically necessary for innovative outcomes that have an encoding that is beyond algorithmic enumeration. Gödel (1931) had seminally used the notion of the Liar, the agent who falsifies or controverts, to embody the pure logic of opposition. However, the Liar can falsify with certainty only from a computable fixed point. This is intuitively well understood in the Lucasian thesis on policy ineffectiveness that regulatees can contravene policy only if the policy outcomes can be rationally expected. When there is mutual recognition of the players of the structure of opposition, the so called fixed point with the Liar can be fully deduced to be a noncomputable fixed point. Any total computable function from this non-computable fixed point referred to as the productive function in Emil Post's set theoretic proof of Gödel incompleteness and as shown in Markose (2001b) to represent the best response function, can only map into a set that cannot be algorithmically enumerated. This coincides with the notion of the strategic use of surprise as intuitively proposed by Lucas (1972). The corresponding equilibrium Type IV dynamics converges to the non-computable domain between recursively inseparable sets is encapsulated in the famous thesis of Langton (1992) on "life at the edge of chaos" and has been set out in Figure 1. The undecidable dynamics implies the logical impossiblility of meta (econometric) models to recursively identify the innovation induced structure change - a matter once again that has been raised in the Lucas Critique (1976).

It was not till the seminal work of Binmore (1987) that game theory even considered the major implications for dynamical systems, as yet not fully understood by economists, of incorporating agents with computational universality and the Gödelian logic involving the Liar or the rule breaker. ${ }^{24}$ A Nash equilibrium in which agents innovate as a best response to evade hostile objectives of other agents and produce novel objects not previously in action sets is currently outside the ambit of traditional game theory. The latter, without the scope of the mathematics of incompleteness, can only consider randomization and not innovation in zero sum and oppositional situations. Markose (2001b) shows that it is indeed the Gödelian logic, the capacity for self-referential calculations by an agent of hostile behaviour of other agents, with the ubiquitous structure of opposition formalized in the Liar, that constitute the necessary

et. al. (1997). At the latter point the immune system wins and a heterogeneous environment for the RNA ceases to exist.

${ }^{24}$ It is my view that it is such irrevocably non-computable structures in strategic situations that Simon (1998) calls the "out guessing problem" which makes the "whole concept of rationality illdefined.."..and .. . "we must adopt some different framework (outside extant game theory) to explain behaviour under these conditions" (ibid. p.188). 
conditions to trigger innovative behaviour with secrecy and subterfuge as being part and parcel of the rational Nash equilibrium strategy at this juncture. In Ray's classic artificial life simulation called Tierra, Ray (1992), in a Red Queen like arms race, when some agents perceive that others are parasitic on them, they start hiding their whereabouts and also mutate to evade the parasite.

\section{Empirical Economics, Power Laws and Other 'Stylized' CAS Facts}

\subsection{The identification problem}

As rightly pointed out by Durluaf, this issue, many of the precursors in the economics literature on CAS, both in the area of economic history and social interaction models have eschewed any explicit connection to complex systems theory either in terms of von Neumann's grand computational foundations or some subset of it as espoused by econo physicists. Nevertheless, we are particularly indebted to eminent economic historians, institutional and evolutionary economists from Hayek, North to Nelson and Winter to wean us off a mathematical economics that is a far cry from the real world complexity.

Despite claims of universality of certain statistical properties of CAS outcomes, in so far as self-organization formally requires non-computability of specific end-states, patterns and equilibria, the significance of the system running its course or path dependent outcomes comes to the forefront. Economic historians and evolutionary economists such as Nelson and Winter (2002, gives a recent overview) have often used their historical case study approach to underscore the point that the problem of path dependence makes the study of processes involved of interest in contrast to end state asymptotic analysis and static efficiency results of neoclassical analysis. Barring Type I and Type II dynamics, the lack of independence from initial conditions and the relevance of the specific time path of processes have to be presumed.

Durlauf bravely tackles the historical accounts on the QWERTY keyboard to address the conceptual issues surrounding path dependence such as whether technological lock-ins are inefficient equilibria or are in fact efficient ones given network effects, fixed costs and so on. It has been argued by Axtell et. al. (2000) on the emergence of equity norms and other path dependent outcomes, that agent models are needed to show in an experimental set up how depending on initial conditions, social systems can manifest meta stable states of inefficient and inequitable social norms that are observed in many societies. A meta stable state is one in which a system spends a very long period of time in terms of intermediate time scales and typically such behaviour of the system cannot be analytically determined, even if as in Axtell et. al. (2000) the asymptotic stochastically stable outcome can be determined a priori.

The bulk of Durlauf's thought provoking contribution on the extant empirical work on complexity in economics surrounds what he calls the identification problem. Inefficient equilibria as he notes can arise from coordination failure as much as from maladaptive lock ins. 
Further, there appears to be no consensus as yet as to what causes power law distributions which have been held up as a manifestation of self-organized critical behaviour.

\subsection{Endogenous Economic Explanations of Power Law}

In many econo physics models, power laws as a stylized CAS fact arising from self-organized criticality, as we saw in previous sections, fall far short of a demonstration of self-organization. This is because the agency for innovation in the system is external to it. Durlauf also raises the problem of the absence of endogenous economically sensible explanations in some econo physics models. However, as already noted even agent based stock market models that have what many would consider to be all the sensible features of neoclassical models, requires external tuning to bring about asset price dynamics that correspond to observed stylized facts to do with fat tails and volatility clustering. The latter are anomalous with the standard Guassian assumptions ${ }^{25}$ on stock returns and neoclassical models on homogenous rational expectations and no trade theorems.

Lux $(2000$,2002) has expended much effort to ascertain the tail index of asset returns in a number of markets. In these studies, Lux finds that values for the tail index for asset returns lies between 2.5 and 4 guaranteeing at least finite first and second moments for the processes. As the latter rule out Levy Stable distributions for asset returns first conjectured by Mandelbroit (1966) and with the statistical rejection of null hypothesis of Guassian/normality for stock returns, it is still an open question as to what class of statistical processes asset returns belong to. Further, there is a large body of alleged evidence for persistence or long memory observable as the hyperbolic decay of the auto correlation function of volatility of asset returns (the absolute or the square of asset returns) (see, Ding et. al.,1993, Breidt et. al., 1998). Lux and Simone(2003) put agent stock market models to good use by finding, after painstaking investigation, that the popular herding framework of Kirman and Teyssiére (2002) which has previously been upheld as an 'explanation' for long memory in asset volatility can only be a spurious result. This is because analytically, the structure of the herding model with two groups of agents, fundamentalists and chartists, follows a bounded Markovian process with a bimodal limiting distribution and "any memory in the system is wiped out by stochastic fluctuations between the two modes of the distribution" (ibid). Nevertheless, standard statistical tests for long memory, when applied to simulated data from this class of agent models, erroneously support the hypothesis for power law decay in the autocorrelation function of the volatility of asset returns. Likewise, Durlauf, this issue, lists a number of shortcomings in the statistical tests of power and scaling laws so far conducted in the literature on markets as CAS.

\footnotetext{
${ }^{25}$ Stock market crashes and other not so extreme events seen in asset markets are anomalous to the Gaussian model of asset returns. In the latter, the 1987 stock market crash with a $22.6 \%$ percent fall in the stock returns in one day is an event that is so improbable that it cannot happen even in the lifetime of the universe, see Sornette (2003, p.50).
} 
A number of econo physicists such as Sornette (1998), Lux and Sornette (2003), Levy and Solomon (1996), Solomon(1998), based on earlier work by Keston (1973) and Goldie (1991), have identified stochastic multiplicative processes ${ }^{26}$ with non-linear lower bound constraints as being contributory to power law distributions in certain economic models. In the Levy and Solomon (1996) artificial stock market model, this lower bound constraint appears for each agent as a condition that his wealth does not fall below the average investor wealth. Milakovic (2001) in a survey on endogenous explanations for the power law exponent close to $3 / 2$ in wealth distribution claims that the condition for power law distribution in the Levy and Solomon (1996) model "depends on an arbitrarily imposed lower bound "constraint on individual agent's wealth defined as a function of the mean wealth of the population. Following recent developments on the Red Queen principle reported in Section 3, Markose et. al.(2003) have argued that this is far from an arbitrary constraint. It can in fact be viewed as the so called Red Queen constraint. The satisfaction of this lower bound constraint is modelled in Markose et. al. (2003) as a behavioural one that arises endogenously from coevolving stock market agents. It then dictates performance enhancement in adaptive learning of investment strategies that will enable agents to 'keep up' with the market Red Queen style. It has been analytically shown by Solomon (1998) that if no attempt is made by those who fail to keep up, then the crucial power law exponent $\alpha$ on wealth distribution (see footnote 12) can fall below 1, signalling a highly inegalitarian wealth distribution. Conversely, if 'too much' effort is put into keeping up, then the $\alpha$ power law exponent will exceed 2, which manifests a more egalitarian wealth distribution than can be warranted by the historically observed $\alpha \sim 3 / 2$. We have seen that in artificial stock market models in which agents have to individually learn and adapt in a multi population GP environment, retraining of GPs is mostly done in an ad hoc way. When the Red Queen constraint was explicitly set up in a multi-population form of constraint enhanced GPs who are the agent/investors in an artificial stock market, Markose et. al. (2003) find some evidence on how the crucial $\alpha \sim 3 / 2$ can emerge in investor wealth distribution. When the Red Queen constraints are not imposed in the artificial stock market, the power law exponent on investor wealth diverge markedly from the required $\alpha \sim 3 / 2$. However, these results are preliminary and it is far from conclusive whether what one might consider to be CAS type agent based features can endogenously in a behavioural sense explain the regularities in the global data.

\subsection{Econometric models of surprises in policy games and the Lucas Critique}

\footnotetext{
${ }^{26}$ A stochastic multiplicative process is one where $\mathrm{x}(\mathrm{t}+1)=\lambda \mathrm{x}(\mathrm{t})$, with $\lambda$ random, even time varying with finite support.
} 
One of the major themes of the computational legacy of CAS as discussed in Sections 3.3 is that the two Lucas (1972) postulates on policy ineffectiveness, the strategic use of surprises and the so called Lucas Critique, Lucas (1976), are stylized CAS facts of fundamental significance.

In the extant policy design literature, the nexus between predictable policy leading to policy/institutional failure and the role of surprise strategies is confined to highly stylised macro economic models on monetary neutrality and surprise inflation. ${ }^{27}$ Remarkably, though macroeconomists in the last three decades made the notion of 'surprise' strategies common currency, at no time was it regarded necessary to establish a generic class of (policy) games where 'surprise' strategies hold in a Nash equilibrium in accordance with the first Lucasian postulate on why policy is rendered ineffective. Hence, the generic necessity of the strategic use of indeterminism due to the presence of hostile agents has not been recognized in the macro policy literature. ${ }^{28}$ Further, the indeterminacy of optimal behavioural structures raised in the problem of lack of structural invariance in the Lucas Critique is regarded to be unrelated to the first two Lucasian postulates.

Some of the econometric issues relating to the Lucas Critique were recently reviewed in Hoover (1995). Indeed, many (see, Ericsson et. al. ,1995, 1996) hold that the applicability of the Lucas Critique on the lack of structural invariance is an empirical question, refutable by the presence of invariance in the system. ${ }^{29}$ Thus, it is not far off the mark to say that it has not been recognized that theory, in particular, CAS theory can throw light on this endogenously arising innovative behaviour of agents as best response strategies in rivalrous oppositional structures which then lead to Type IV undecidable dynamics of Figure 1.

With no theoretical guidance as to which class of games warrant elimination of extant policy rules/institutions and the adoption of innovative or surprise strategies in the Nash equilibrium signalling structural breaks in the system and therefore to predictive failure with meta forecast models, Ericsson and Irons (1995) found "exactly zero papers (that yield) empirical support for the Lucas Critique" after an extensive survey of the empirical literature,

\footnotetext{
${ }^{27}$ The notion of a surprise strategy in the economics literature appears in the so called Lucas surprise supply function often defined as follows: $y=y^{*}+b\left(\pi-\pi^{e}\right)+\varepsilon$. This says that output, $y$, will not increase beyond the natural rate, $y^{*}$, unless there is 'surprise' inflation, $\left(\pi-\pi^{e}\right)$ which is the white noise prediction error from expected inflation, $\pi{ }^{\mathrm{e}}$. The idea here is that the private sector contravenes the effects of anticipated inflation, viz. the neutrality result. Hence, it is intuitively asserted that authorities who seek to expand output beyond the natural rate need to use surprise inflation.

${ }^{28}$ The attempts by macro policy theorists to eliminate 'surprise' inflation and indeterminism by using a transparent precommitment strategy in the form of the currency peg led to some of the worst policy disasters with the serial collapse of pegged systems in the last decade, Eichengreen (1999). Soros ( 1995) allegedly claimed that he used the Liar strategy to profit from the collapse of the British currency peg in the summer of 1992. None in the macro economics fraternity saw the relevance of Binmore (1987) who raised the spectre of Gödel and hence the significance of the allusion to the Liar / hostile agent who will bring about the demise of institutions that are based on strategies that have predictable outcomes.

${ }^{29} \mathrm{I}$ 'm grateful to L.Ellis from the Reserve Bank of Australia for bringing this to my attention.
} 
Leeper (1985,p.314). Finally, it must be noted, adaptive agent models of Column III, Table I are methodologically better suited to study unintended consequences of changes in policy rules as they are designed to manifest coevolution and do not run into the Lucas Critique of econometric models.

\section{Concluding Remarks}

Following the von Neumann computational legacy on CAS which is based on mathematical advances in the areas of effective computation or procedures, incompleteness and dynamical systems theory, the major hypothesis emanating from Wolfram (1984), Langton (1990, 1992), Kaufmann (1993) and Casti (1994) is that the sine qua non of complex adaptive systems is their capacity to produce novelty or 'surprises' and the so called Type IV innovation based structure changing dynamics of the Wolfram-Chomsky schema. Despite the deep mathematical foundations of CAS, remarkably, systems capable of adaptive novelty are commonplace and only involve the intuitively familiar notion on the need to evade hostile agents. Axelrod (1987) in his classic study on cooperative and non-cooperative behaviour in governing design principles behind evolution raised the crucial question on the necessity of hostile agents :" we can begin asking about whether parasites are inherent to all complex systems, or merely the outcome of the way biological systems have happened to evolve" (ibid. p. 41). Based on the Gödel (1931) incompleteness result, in addition to the formal structure of opposition as being a logically necessary condition for evasive and innovative behaviour, Wolfram (1984) had conjectured that the highest level of computational intelligence, the capacity for self-referential calculation of hostile behaviour was also necessary. The role of economists in the multi disciplinary edifice of CAS, especially their contribution to the crucial insights and ingredients of CAS theory on the strategic use of surprises and indeterminacy as part of successful evolutionary design principles- is not insubstantial.

Mathematically, problems involving strongly self-referential global/ system wide mappings or those that involve coevolutionary contrarian or hostile agents make these impossible to be solved by deductive means of Column I, Table 1. Adaptive/evolutionary methods of Column III, Table 1 have to be used and models beyond optimization and the rational economic man of neoclassical economics are needed. The algorithmic unsolvability of the fixed point mappings of equilibria with adaptive novelty in the Gödelian structure (see, Figure 1), makes self-organization the flip side of CAS and is perhaps the unifying principle for all complex systems. It is increasingly being understood, as explained in Sections 1.2.1 and 3.3, that the seminal Bak notion of self-organized critical systems with large numbers of interacting constituent parts that sustain a critical degree of variety or heterogeneity, requires an endogenous dynamic currently being referred to as the Red Queen principle. The latter can be viewed to govern the rate of performance enhancement and the rate of innovative activity 
among coevolving competitive agents. There is however, no consensus as to whether it is the Red Queen principle and/or some other endogenous causal factor such as the diffusion of information in networked systems that gives rise to the power laws associated with SOC systems. Indeed, as we have noted, many socio dynamic models have eschewed any CAS lineage as we have yet to fully understand the notion of self-organization with a critical degree of heterogeneity to supplant homogeneity and also the universality principles on the network connectivity in agents to maintain criticality.

The new computational CAS paradigm clearly surpasses the traditional evolutionary Darwinian framework of random mutation, heredity and natural selection. The principles of self-organization outlined in this overview go well beyond the Darwinian ideas of natural selection currently operationalized in replicator dynamics. Crucially, the formal results that agents with the highest level of computational intelligence are needed to produce adaptive novelty with empirical evidence of the Red Queen dynamic in immunology, in product innovation and regulatory arbitrage in the capitalist system, has cast doubt on random mutation as being the only source of variety. The equilibrium with self-organized complexity with adaptive novelty of Figure 1 has the highest degree of algorithmic unsolvability of the Hilbert Tenth problem. This model of indeterminism is a far cry from extant models that appear to assume adaptive innovation or strategic 'surprise' (see, footnote 27) as white noise which in the framework of entropy represents perfect disorder, the antithesis of self-organized complexity. It can be conjectured that a lack of progress in our understanding of market incompleteness and arbitrage free institutions is related to these issues on indeterminism.

This Feature on markets as CAS has been critical of some multi-disciplinary perspectives of complex systems theory to Economics. It is also original in highlighting the computational foundations of CAS theory and the significance of the Red Queen dynamic in the shaping of macro evolutionary trends and in the process of self-organization that governs the critical degree of variety or heterogeneity in systems. In conclusion, methodologically the view of CAS as a novelty producing system with attendant problems of incompleteness spells a paradigm shift for many mainstream economists who have operated under the assumption of closure and completeness.

\section{References}

Abramson, G., and Kuperman, M. (2001). 'Social Games in a Social Network', Physics Review, E. Vol. 63.

Ackley, D.H and Litterman M. (1992). 'Interactions Between Learning and Evolution', In C.G Langton, C. Taylor, D. Farmer and S. Rasmussen (Eds.), Artificial Life II, Santa Fe Institute Studies in the Sciences of Complexity, Vol. 10, Addison Wesley.

Albin, P. (1998). Barriers and Bounds to Rationality, Essays on Economic Complexity and Dynamics in Interactive Systems, Edited and with an Introduction by D. Foley, Princeton University Press. 
Anderson, P. (1977). 'Local Moments and Localized States', Nobel Lecture http://www.nobel.se/physics/laureates/1977/anderson-lecture.pdf

Arenas, A., DL' az-Guilera A., Perez C.J., Vega-Redondo F. (2002). 'Self-Organized Criticality in Evolutionary Systems With Local Interaction', Journal of Economic Dynamics and Control, vol. 26, pp.2115-2142

Arifovic, J. (1994). 'Genetic Algorithm Learning and the Cobweb Model', Journal of Economic Dynamics and Control, vol.18, pp.3-28.

Arifovic, J.(2003), 'Evolutionary Algorithms in Macroeconomic Models', Macroeconomic Dynamics, vol. 4, pp. 373-414.

Arrow, K.J and F.H Hahn (1971). General Competitive Analysis, Amsterdam, North Holland .

Arthur, W.B. (1991). 'Designing Economic Agents that Act Like Human Agents: A Behavioural Approach To Bounded Rationality', American Economic Review, 81, 353-359.

Arthur, W.B. (1994). 'Inductive Behaviour and Bounded Rationality', American Economic Review, 84, pp.406-411.

Arthur, W.B, Holland, J., Le Baron, B., Palmer, R., Taylor, P. (1997). 'Asset Pricing Under Endogenous Expectations in an Artificial Stock Market', In Arthur W.B., Durlauf S., Lane, D. (Eds) The Economy as an Evolving Complex System II, Addison Wesley, pp. 15-44.

Axelrod, R. (1984). The Evolution of Cooperation, Basic Books, New York.

Axelrod, R. (1987). 'The Evolution of Strategies in the Iterated Prisoner's Dilemma', in Davis, L. (ed.) Genetic Algorithms and Simulated Annealing, Research Notes in AI, Pitman/Morgan Kaufmann, pp. 32-41.

Axtell, R., Epstein, J., Young, P. (2002). 'The Emergence of Classes in a Multi-Agent Bargaining Model', Working Paper No. 9, Center for Social and Economic Dynamics.

Bak, P. Tang, Wiesenfeld, C. (1988). 'Self-organized Criticality', Physical Review A, 38, 364- 373.

Bak, P. and Sneppen, K. (1993). 'Punctuated Equilibrium and Criticality in a Simple Model of Evolution ', Physical Review Letters 74, 4083-4086.

Bak, P.(1996). How Nature Works:The Science of Self-Organized Criticality, Springer- Verlag(Copernicus), New York.

Binmore, K.(1987). 'Modelling Rational Players: Part 1', Journal of Economics and Philosophy, 3, 179-214.

Binmore, K. (1998). Game Theory and the Social Contract II, Just Playing, MIT Press.

Blume, L. (1993). 'The Statistical Mechanics of Strategic Interaction', Games and Economic Behaviour, 4, 387-424.

Breidt, F.J., N. Crato and P. de Lima (1998). 'The Detection and Estimation of Long Memory in Stochastic Volatility', Journal of Econometrics, 83, pp.325-348.

Casti, J. (1994). Complexification : Explaining A Paradoxical World Through the Science of Surprises, London Harper Collins.

Challet, D. and ZhangY.C. (1998). 'On The Minority Game: Analytical And Numerical Studies', Physica, 256,514.

Chen Shu-Heng, and Yeh C-H. (2001). 'Evolving Traders and the Business School with Genetic Programming: A New Architecture of the Agent-Based Artificial Stock Market', Journal of Economic Dynamics and Control, 25, pp.363-393.

Chwe, M., (2000). 'Communication and Coordination in Social Networks', Review of Economic Studies, 67, 1-16.

Cutland, N.J.(1980). Computability: An Introduction to Recursive Function Theory, Cambridge University Press.

Day, R. and Chen,P. (993). Edited, Non Linear Dynamics and Evolutionary Economics, Oxford University Press.

Dawid, H. (1999). Adaptive Learning by Genetic Algorithms : Analytical Results and 
Applications to Economic Models, $2^{\text {nd }}$. Edition, Springer.

Durlauf, S., and Peyton Y. (2000). Social Dynamics, The Brookings Institution, Washington, D.C.

Dosi, G. and Nelson,R. (1994). 'An Introduction to Evolutionary Theories in Economics', Journal of Evolutionary Economics , 4, pp.153-172.

Ding, Z., Granger C.W.J. and Engle R.F. (1993). 'A Long Memory Property of Stock Market Returns and a New Model', Journal of Empirical Finance 1, 83-106.

Epstein, L., and Axtell, R., 1996, Growing Artificial Societies: Social Science from Bottom Up, MIT Press and Brookings Institution Press.

Ericsson, N. and Irons, J.(1995). 'The Lucas Critique in Practice: Theory Without Measurement' Chapter 8 in, Macroeconometrics:Developments, Tensions and Prospects, ed. K.D. Hoover, Kluver Academic Publishers.

Ericsson, N., Hendry, D., Mizon, G.(1996). 'Exogeniety, Cointegration and Economic Policy Analysis', Board of Governors of Federal Reserve System, International Finance, Discussion Paper, no. 616.

Eichengreen, B. (1999), 'Kicking The Habit: Moving From Pegged Rates To Greater Exchange Rate Flexibility', Economic Journal, vol. 109, pp.C1-C15.

Foster, D., and Young, P., 1990, 'Stochastic Evolutionary Game Dynamics', Theoretical Population Biology, 38, pp. 219-232.

Gode, D.K and Sunder, S., (1993). 'Allocative Efficiency of Markets With Zero Intelligence Traders : Markets as a Partial Substitute for Individual Rationality', Journal of Political Economy, 101, 119-137.

Gödel, K. (1931). 'On Formally Undecidable Propositions of Principia Mathematica and Related Systems '(Translation in English in Gödel's Theorem in Focus, ed. .S.G Shanker, 1988, Croom Helm).

Goldberg, D.E.(1989). Genetic Algorithms in Search, Optimization and Machine Learning, Reading, M.A, Addison Wesley.

Goldberg, D.E, (1995). "The Existential Pleasures of Genetic Algorithms , in G. Winter, J. Perioux, M. Galan and P. Cuesta (Eds), Genetic Algorithms in Engineering and Computer Science, pp. 23-31, Wiley and Sons.

Goldie, C. (1991). 'Implicit Renewal Theory and Tails of Solutions of Random Equations', Annals of Applied Probability 1, 126-66.

Goyal, S. and Janssen, M. (1996). 'Interaction Structure and Social Change', Journal Of Institutional and Theoretical Economics, 152, 472-495.

Green, D. (1994). 'Emergent Behaviour in Biological Systems', Complexity International , 1, 1-12.

Hayek, F.A., (1945). 'The Use of Knowledge in Society', American Economic Review, 35, 519-530.

Hayek, F.A.,(1967). Studies in Philosophy, Politics and Economics, Simon and Schuster,N.Y. Hayek, F. A.(1952). The Sensory Order, University of Chicago Press, Chicago, IL.

Hayek, F.A., (1982). The Sensory Order After 25 Years, in: W.B. Weimar and D.S. Palermo, eds., Cognition and Symbolic Processes, Vol.2, Lea Publishers, N.J.

Hemelrijk, C.K. (1997) “Cooperation without Genes, Games or Cognition”. In P.H Harvey(Ed.) Fourth European Conference on Artificial Life, (pp.511-520). Cambridge: MIT Press.

Hillis, W.D. (1992). 'Co-Evolving Parasites Improve Simulated Evolution as an Optimization Procedure', In Langton, C., Taylor, J. Doyne Farmer, and S, Rasmussen (Eds.) Artificial Life II (Sante Fe Institute Studies in the Sciences of Complexity, Vol 10) Reading MA : Addison-Wesley.

Ho, M.W., and Saunders, P.T. (1986). 'Evolution: Natural Selection Or Self-Organization ?', In C.W. Kilmister (Ed.) Disequilibrium and Self-Organization, D. Reidel Publishing Company.

Hodgson, G., (1993). Economics and Evolution: Bringing Life Back Into Economics , Basil Blackwell, Oxford.

Holland, J.H.(1975,1992). Adaptation in Natural and Artificial Systems, An 
Introductory Analysis with Applications to Biology, Control and Artificial Intelligence $\left(2^{\text {nd }}\right.$. ed.) Cambridge, M.A: MIT Press.

Holland, J.H. (1992). 'Complex Adaptive Systems', Daedalus, 121, 17-20.

Holland, J.H., (1998). Emergence: From Chaos to Order, Addison Wesley, Longman.

Hoover, K.D.(1995): (Edited by).Macroeconometrics:Developments, Tensions and

Prospects, Kluwer Academic Publishers.

Jackson, M. and Watts, A. (2001). 'The Evolution of Social and Economic Networks', Forthcoming in the Journal of Economic Theory.

Jensen, H. (1998). Self-Organized Criticality, Cambridge University Press.

Keller, E.F. and Segel,L.(1970). 'Initiation of Slime Mold Aggregation Viewed as an Instability', Journal of Theoretical Biology, vol. 26, pp 399-415.

Keston, H. (1973). 'Random Difference Equations and Renewal Theory for Products of Random Matrices', Acta Mathematica 131, 207-48.

Kauffman, S.A (1993). The Origins of Order: Self Organization and Selection in Evolution, Oxford University Press.

Kirman, A. and Teyssiere, G. (2002). 'Microeconomic Models for Long-Memory in the Volatility of Financial Time Series', Studies in Nonlinear Dynamics and Econometrics, 5,

Krugman, P. (1996). 'The Self-Organizing Economy', Blackwell.

Leeper, E.M.,(1995). 'Commentary on Chapter 8', Macroeconometrics:

Developments, Tensions and Prospects, Ed. K.D. Hoover, Kluver Academic Publishers.

Ligett, T.M.(1985). Interacting Particle Systems (Springer-Verlag, New York).

Ligett,T.M. (1997). 'Stochastic Models of Interacting Systems' . The Annals of Probability, 25,1-29.

Langton, C.(1990). 'Computation at the Edge of Chaos: Phase Transitions and Emergent Computation', in Emergent Computation, Ed. By S. Forrest, North Holland.

Langton, C.(1992). 'Life at the Edge of Chaos', In C. Langton, C. Taylor, J. Doyne Farmer, and S, Rasmussen (Eds.) Artificial Life II (Sante Fe Institute Studies in the Sciences of Complexity, Vol 10) Reading MA : Addison-Wesley.

Levy, M., and Solomon S. (1996). 'Dynamical Explanation For the Emergence of Power Law in a Stock Market Model', International Journal of Modern Physics C, 7, 65-72.

Lewis, A.A., (1985). 'On Effectively Computable Choice Functions', Mathematical Social Sciences, 10.

Lewis, A.A. (1987). 'On Turing Degrees of Walrasian models and a General Impossibility Result in the Theory of Decision Making', Technical Report no. 512 (Centre for Research for Organization Efficiency, Stanford University).

Lucas, R.(1972). ' Expectations and the Neutrality of Money', Journal of Economic Theory, 4, pp.103-24.

Lucas, R.(1976). 'Econometric Policy Evaluation: A Critique', Carnegie-Rochester Conference Series on Public Policy, vol. 1, pp.19-46.

Lux, T. and Marchesi M. (1999). 'Scaling and Criticality In Stochastic Multi-Agent Model of a Financial Market', Nature, vol. 397, pp. 498-500.

Lux, T. (2000). 'On Moment Condition Failure in German Stock Returns: and Application Of Recent Advances in Extreme Value Statistics', Empirical Economics, 25, 641652.

Lux, T., and Ausloos M.(2002). 'Market Fluctuations I: Scaling, Multiscaling and their Possible Origins ', In, ed. A. Bunde, J.Kropp, and H.J Schellnhuber, Theories of Disaster - Scaling Laws Governing Weather, Body and Stock Market Dynamics, 373-409.

Lux, T. and D. Sornette (2002). 'On Rational Bubbles and Fat Tails', Journal of Money, Credit and Banking, vol.34, pp 589-610.

Mailath, G., Samuelson, L, and, Shaked, A. (1997). 'Endogenous Interactions', in U. 
Pagano and N. Nicita, eds., The Evolution of Economic Diversity, Routledge.

Mandelbroit, B.( 1966). 'When Can Price Be Arbitraged Efficiently? A Limit to The Validity of the Random Walk and Martingale Models', The Review of Economics and Statistics, 225-236.

Markose, S.(2001a), Book Review of Computable Economics by K. Velupillai, In The Economic Journal, vol. 111, pp.468- 484.

Markose, S. (2001b), "The Liar and Surprises: So What Is The Lucas Critique- A New Perspective From Computability Theory", University of Essex, Economics Department, Mimeo, http//www.essex.ac.uk/economics/staff/scher.html

Markose, S. (2002). 'The New Evolutionary Computational Paradigm of Complex Adaptive Systems: Challenges and Prospects For Economics and Finance', In Genetic Algorithms and Genetic Programming in Computational Finance, Edited by Shu-Heng Chen, Kluwer Academic Publishers. Also Essex University Economics Discussion Paper no. 552.

Markose, S., Tsang, T. and Martinez, S. (2003). 'The Red Queen Principle and the Emergence of Efficient Financial Markets : An Agent Based Approach', mimeo presented at $8^{\text {th }}$ Workshop on Economics and Heterogeneous Interacting Agents (WEHIA), Kiel.

Marimon, R., Mc Gratton E., and Sargent, T.J, (1989). 'Money as a Medium of Exchange in an Economy with Artificially Intelligent Agents', Journal of Economic Dynamics and Control, 14, 329-373.

Menger, C. (1892) 'On the Origin of Money', The Economic Journal, vol.2, pp.238255.

Miller, M. (1986). 'Financial Innovation : The Last Twenty Years and the Next', Journal of Financial and Quantitative Analysis, vol. 21, pp. 459-471.

Milakovic, M. (2001). 'A Statistical Equilibrium Model of Wealth Distribution', mimeo, Center for Economic Policy Analysis, New School University.

Mirowski, P. (2000). Machine Dreams: How Economics Became a Cyborg Science. Cambridge University Press, New York.

Morris, S. (2000). 'Contagion', Review of Economic Studies, vol. 67, pp.57-78.

Nelson, R.R., and S.G. Winter (2002). 'Evolutionary Theorizing in Economics', Journal of Economic Perspectives, vol. 16, no.2, pp.23-46.

Nicolis, G. and Prigogine, I. (1977) Self-Organization in Non-Equilibrium Systems, Wiley, New York.

Nicolis, G. and I. Prigogine (1989). Exploring Complexity : An Introduction, Freeman, New York.

Papadimitriou, C.H. (1994). 'On the Complexity of the Parity Argument and Other Inefficient Proofs of Existence', Journal of Computer and System Sciences, 48, pp. 498-532.

Pareto, V., 1897, Cours d'Economique Politique, Vol.2, Macmillan, London.

Penrose, R. (1988). 'On Physics and Mathematics of Thought', In The Universal Turing Machine: A Half Century Survey, Edited by Rolf Herken, Oxford University Press.

Post, E.(1944). 'Recursively Enumerable Sets of Positive Integers and Their Decision Problems', Bulletin of American Mathematical Society, vol.50, pp.284-316.

Ray, T.S. (1992). 'An Approach to The Synthesis of Life', In C. Langton, C. Taylor, J. D.Farmer, and S, Rasmussen (Eds.) Artificial Life II (Sante Fe Institute) Addison-Wesley.

Robson, A.(2002). 'The Evolution of Rationality and the Red Queen', Journal of Economic Theory, vol.111, pp.1-22.

Rust, J. (1998). 'Dealing With the Complexity of Economic Calculations', In S. Durlauf and J. Traub, eds. Limits to Knowledge in Economics. Addison-Wesley.

Schelling, T. (1978). Micromotives and Macrobehaviour, Nortin, New York, N.Y.

Schumpeter, J.(1950). Capitalism, Socialism and Democracy, New York: Harper and Row. 
Simon, H.A.(1956), 'Rational Choice and the Structure of the Environment', in Models of Thought, Yale University Press, New Haven, 1979.

Simon, H.A. (1976). 'From Substantive to Procedural Rationality', in S.J. Latsis, ed. Methods and Appraisal in Economics, 129-148, Cambridge University Press.

Simon, H.A. (1998). 'Simon's Critique', In, Modelling Bounded Rationality, Ariel Rubinstein, MIT Press.

Sims, K.(1994),'Evolving 3-D Morphology and Behaviour by Competition', In Brooks, R. and Maes, P. (Edited), Proceedings of Fourth Workshop on Artificial Life, pp.28-39, Boston, MA., MIT Press.

Solé, R., Ferrer, R., Gozalez-Garcia, I., Quer, J., Domingo, E., 1997 ‘The Red Queen Dynamics, Competition and Critical Points in a Model of RNA Virus Quasispecies', Santa Fe Institute Discussion Paper No.97-12-086.

Solomon, S. (1998), 'Stochastic Lotka-Volterra Systems of Competing AutoCatalytic Agents Lead Generically To Truncated Pareto Wealth Distribution, Truncated Levy Distribution of Market Returns, Clustered Volatility, Booms and Crashes', In, Computational Finance, 97, Eds. A.P.N Refenes, A.N Burgess, J.E Moody, Kluwer Academic Publishers.

Sornette, D. (1998). 'Multiplicative Processes and Power Laws' Physical Review E 57, 4811-13.

Sornette,D.(2003), 'Why Stock Markets Crash ?', Princeton University Press.

Spear, S.(1989). "Learning Rational Expectations Under Computability Constraints", Econometrica, 57, pp.889-910.

Soros, G.(1995). Soros on Soros: Staying Ahead of the Curve, John Wiley.

Sunder, S.(2000), 'Market as an Artifact: Aggregate Efficiency from Zero Intelligence Traders', Working Paper No. 02-16, Yale International Center for Finance.

Testfasion, L.( 1998). ACE website.

Tsang, E., Jin, N., and Gosling, T. (2003). 'Repeated Prisoner's Dilemma in Evolutionary Computation : A Rigorous Re-examination ', Mimeo Department of Computer Science and Institute For Studies in Finance, University of Essex, http:/www.cs.adfa.edu.au/cec_2003/index.html

Turing, A. (1936). "On Computable Numbers With an Application To the Entscheidungsproblem ” In M. Davis, (ed.) The Undecidable, Raven, New York.

van Valen, L. (1973). ‘A New Evolutionary Law', Evolutionary Theory, 1, 1-30.

Velupillai, K. (2000), Computable Economics, Arne Ryde Lectures, Oxford University Press.

von Neumann, J. (1970). Theory of Self-Reproducing Automata, ed. Burks, A.W, Urbana: University of Ilinois Press.

Watts, D.J., Strogatz, S.H.(1998). 'Collective Dynamics of 'Small-World' Networks', Nature, vol. 393, pp. 440-442.

Watts, D. (1999). Small Worlds, Princeton University Press.

Wolfram, S. (1984). 'Cellular Automata As Models of Complexity”, Physica, 10D,135. 